\title{
Computation of compressible quasi-axisymmetric slender vortex flow and breakdown
}

\author{
Osama A. Kandil ${ }^{1}$ and Hamdy A. Kandil ${ }^{2}$ \\ Department of Mechanical Engineering and Mechanics, Old Dominion University, Norfolk, VA 23529-0247, USA
}

\begin{abstract}
Analysis and computation of steady, compressible, quasi-axisymmetric flow of an isolated. slender vortex are considered. The compressible Navier-Stokes equations are reduced to a simpler set by using the slenderness and quasi-axisymmetry assumptions. The resulting set along with a compatibility equation are transformed from the diverging physical domain to a rectangular computational domain. Solving for a compatible set of initial profiles and specifying a compatible set of boundary conditions, the equations are solved using a type-differencing scheme. Vortex breakdown locations are detected by the failure of the scheme to converge. Computational examples include isolated vortex flows at different Mach numbers, external axial-pressure gradients and swirl ratios. Excellent agreement is shown for a bench-mark case between the computed results using the slender vortex equations and those of a full Navier-Stokes solver.
\end{abstract}


$=$

$\square$

$\bar{\equiv}$
$\overline{\overline{\underline{\equiv}}}$

$\underline{\underline{m}}$

$\bar{E}$

$\overline{=}$

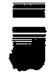

$\overline{\bar{F}}$

$=$

-

豆

$\overline{\overline{\underline{\underline{\underline{\underline{ }}}}}}$

晋

$\bar{\equiv}$

$-$

$\overline{-}$

를

景

$\underline{\equiv}$

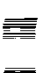




\title{
Computation of compressible quasi-axisymmetric slender vortex flow and breakdown
}

\author{
Osama A. Kandil ' ${ }^{2}$ and Hamdy A. Kandil ${ }^{2}$ \\ Department of Mechanical Engineering and Mechanics, Old Dominion Únitersity, Norfolk, VA 23529.0247, USA
}

\begin{abstract}
Analysis and computation of steady, compressible, quasi-axisymmetric now of an isolated. slender vortex are considered. The compressible Navier-Stokes equations are reduced to a simpler set by using the slenderness and quasi-axisymmetry assumptions. The resulting set along with a compatibility equation are transformed from the diverging physical domain to a rectangular computational domain. Solving for a compatible set of initial profiles and specifying a compatible set of boundary conditions, the equations are solved using a type-differencing scheme. Vortex breakdown locations are detected by the failure of the scheme to converge. Computational examples include isolated vortex flows at different Mach numbers, external axial-pressure gradients and swirl ratios. Excellent agreement is shown for a bench-mark case between the computed results using the slender vortex equations and those of a full Navier-Stokes solver.
\end{abstract}

\section{Introduction}

The phenomenon of vortex breakdown or bursting was observed in the water vapor condensation trails along the leading-edge vortex cores of a gothic wing. Two forms of the leading-edge vortex breakdown, a bubble type and a spiral type, have been documented experimentally [1]. The bubble type shows an almost axisymmetric sudden swelling of the core into a bubble, and the spiral type shows an asymmetric spiral filament followed by a rapidly spreading turbulent flow. Both types are characterized by an axial stagnation point and a limited region of reversed axial flow. Much of our knowledge of vortex breakdown has been obtained from experimental studies in tubes where both types of breakdown and other types as well were generated [2-4].

The major effort of numerical simulation of vortex breakdown flows has been focused on incompressible, quasi-axisymmetric isolated vortices. Grabowski and Berger [5] used the incompressible, quasi-axisymmetric Navier-Stokes equations. Ha-

\footnotetext{
1 Professor and Eminent Scholar.

2 Graduate Research Assistant.
}

fez et. al [6] solved the incompressible, steady, quasi-axisymmetric Euler and Navier-Stokes equations using the stream function-vorticity formulation and predicted vortex breakdown flows similar to those of Garbowski and Berger. Spall, Gatski and Grosch [7] used the vorticity-velocity formulation to solve the three-dimensional. incompressible, unsteady Navier-Stokes equations.

Flows around highly swept wings and slender wing-body configurations at transsonic and supersonic speeds and at moderate to high angles of attack are characterized by vortical regions and shock waves, which interact with each other. Other applications which encounter vortex-shock interaction include a supersonic inlet ingesting a vortex and injection into a supersonic combustor to enhance the mixing process, see Delery et. al [8] and Metwally, Settles and Horstman [9]. These problems and others call for developing computational schemes to predict, study and control compressible vortex flows and their interaction with shock waves. Unfortunately, the literature lacks this type of analysis with the exception of the preliminary work of Liu, Krause and Menne [10] and Copening and Anderson [11].

In this paper, the steady, compressible NavierStokes equations are simplified using the quasi- 


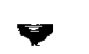

$\bar{\nabla}$

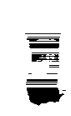

$\equiv$

$\equiv$

$=$

$\square$

$=$

를

$=$

i

䇾

衰

E

흘

I

$\underline{\underline{\underline{\underline{\underline{\underline{\underline{E}}}}}}}$

틀

$\underline{\underline{\underline{\underline{\underline{E}}}}}$

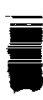

! 
axisymmetry and slenderness assumptions. A compatibility equation [10] has been used and the governing equations are transformed to a rectangular computational domain by using a LeveyLee-type transformation. A compatible set of initial conditions and boundary conditions is obtained and the problem is solved using a type-differencing scheme. The numerical results show the effects of compressibility, external axial pressure gradients and the swirl ratio on the vortex breakdown location. A bench-mark flow case has been solved using these equations and the full NavierStokes equations. The results are in excellent agreement with each other.

\section{Highlights of the formulation and computational scheme}

Starting with the steady, compressible NavierStokes equations which are expressed in the cylindrical coordinates $(\tilde{x}, \tilde{r}$ and $\phi)$, assuming the isolated vortex flow to be slender $[\tilde{r} / l=0$ $(1 / \sqrt{R e}), \tilde{v} / U_{\infty}=\mathrm{O}(1 / \sqrt{\operatorname{Re}})$, where $l$ is a characteristic length, $\tilde{v}$ the radial velocity, $U_{\infty}$ the freestream velocity and $\operatorname{Re}$ the freestream Reynolds number] and quasi-axisymmetric $[\partial / \partial \phi()=$ $0]$, and performing an order-of-magnitude analysis, the equations are reduced to a compressible, quasi-axisymmetric, boundary-layer-like set. The dimensionless flow variables $\rho, p, u, v, w, T$ and $\mu$ are non-dimensionalized by $\rho_{\infty}, \rho_{\infty} a_{\infty}^{2}, a_{\infty}$, $a_{\infty}^{2} / C_{\rho}$ and $\mu_{\infty}$ for the density, pressure, velocity, temperature and viscosity, respectively, where $C_{p}$ is the specific heat at constant pressure. Next, we introduce a Levey-Lee-type transformation which is given by

$\xi=\int_{0}^{x} \rho_{\mathrm{e}} \mu_{\mathrm{e}} \mathrm{d} x, \quad \eta=\frac{\rho_{\mathrm{e}}}{\lambda(\xi)} \int_{0}^{r} \frac{\rho}{\rho_{\mathrm{e}}} \mathrm{d} r$,

where $\lambda$ is given by

$$
\begin{aligned}
\text { MSF }= & \frac{\lambda(\xi)}{f(\rho)}=\frac{r_{\mathrm{e}}(\xi)}{r_{\mathrm{e}}\left(\xi_{\mathrm{i}}\right)} \\
\equiv & \text { modified shape factor characterizing } \\
& \text { the growth of vortex-flow boundary }
\end{aligned}
$$

and $f(\rho)$ is a function relating the density integral at any axial station to that at the initial station. It is equal to 1 for incompressible flow. The subscript e refers to external conditions and the subscript i refers to initial location.

The governing equations become

$$
\frac{\partial V}{\partial \eta}+\frac{1}{r \lambda} \frac{\partial}{\partial \xi}(\lambda u r)+\frac{\lambda}{\rho r} V=0,
$$

where $v=\frac{\rho_{\mathrm{e}} \mu_{\mathrm{e}} \lambda}{\rho} V-\eta_{\mathrm{x}} \frac{\lambda u}{\rho}$, and $\eta_{\mathrm{x}}=\frac{\partial \eta}{\partial x}$,

$$
\begin{aligned}
u \frac{\partial u}{\partial \xi}+V \frac{\partial u}{\partial \eta}= & -\frac{1}{\rho} \frac{\partial p}{\partial \xi}-\frac{\lambda}{\rho} \theta \frac{w^{2}}{r} \\
& +\frac{M}{\lambda r} \frac{\partial}{\partial \eta}\left(\frac{c r}{\lambda} \frac{\partial u}{\partial \eta}\right),
\end{aligned}
$$

where

$$
\begin{aligned}
& \theta=\frac{1}{\rho_{\mathrm{e}} \mu_{\mathrm{e}}} \eta_{x} \quad \text { and } \quad c=\frac{\rho \mu}{\rho_{\mathrm{e}} \mu_{\mathrm{e}}}, \\
& \frac{\lambda}{r} w^{2}=\frac{\partial p}{\partial \eta}, \\
& u \frac{\partial w}{\partial \xi}+V \frac{\partial w}{\partial \eta}+\frac{\lambda}{\rho r}(V-\theta u) w \\
& =\frac{M}{\lambda^{2} r^{2}} \frac{\partial}{\partial \eta}\left[c r^{3} \frac{\partial}{\partial \eta}\left(\frac{w}{r}\right)\right], \\
& u \frac{\partial T}{\partial \xi}+V \frac{\partial T}{\partial \eta} \\
& =\frac{u}{\rho} \frac{\partial p}{\partial \xi}+\frac{\lambda}{\rho} \frac{V w^{2}}{r}+\frac{M}{\operatorname{Pr} \lambda^{2} r} \frac{\partial}{\partial \eta}\left(\operatorname{cr} \frac{\partial T}{\partial \eta}\right) \\
& +\frac{M c}{\lambda^{2}}\left\{\left(\frac{\partial u}{\partial \eta}\right)^{2}+\left[r \frac{\partial}{\partial \eta}\left(\frac{w}{r}\right)\right]^{2}\right\},
\end{aligned}
$$

where $\operatorname{Pr} \equiv$ Prandtl number $=0.72$.

$p=\frac{\gamma-1}{\gamma} \rho T$,

where $\gamma \equiv$ ratio of specific heats.

The viscosity $\mu$ is related to the temperature through the Sutherland law. At the initial boundary, $\xi=\xi_{i}$, we specify

$u_{\mathrm{i}}=u(\eta), \quad w_{\mathrm{i}}=w(\eta)$ and $T_{\mathrm{i}}=T(\eta)$.

The other compatible initial conditions are obtained from a compatibility equation and eqs. (5) 
and (8). At the vortex axis, $\eta=0$, we specify

$\frac{\partial u}{\partial \eta}=V=w=\frac{\partial T}{\partial \eta}=0$

At the outer boundary, $\eta=\eta_{e}$, we assume the boundary to be a stream surface, specify the axial
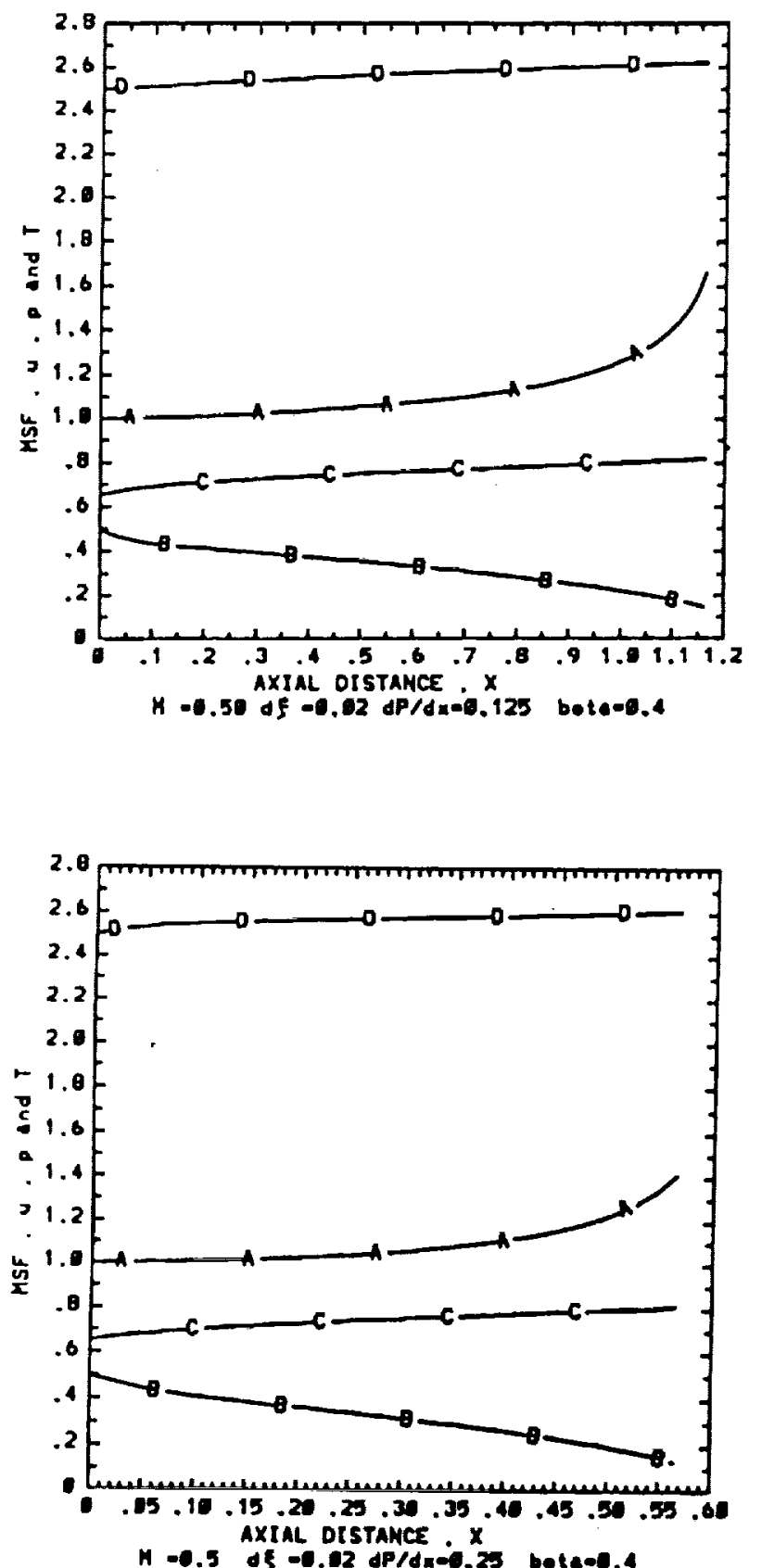

pressure gradient $(\partial p / \partial \xi)_{\mathrm{e}}$ and use the Euler equations to match the outer profiles to those of the viscous core to obtain the conditions on $u_{e}$, $w_{\mathrm{e}}, T_{\mathrm{e}}, \rho_{\mathrm{e}}$.

Eqs. (3)-(7) are solved using an implicit, typedifferencing scheme. The computational proce-
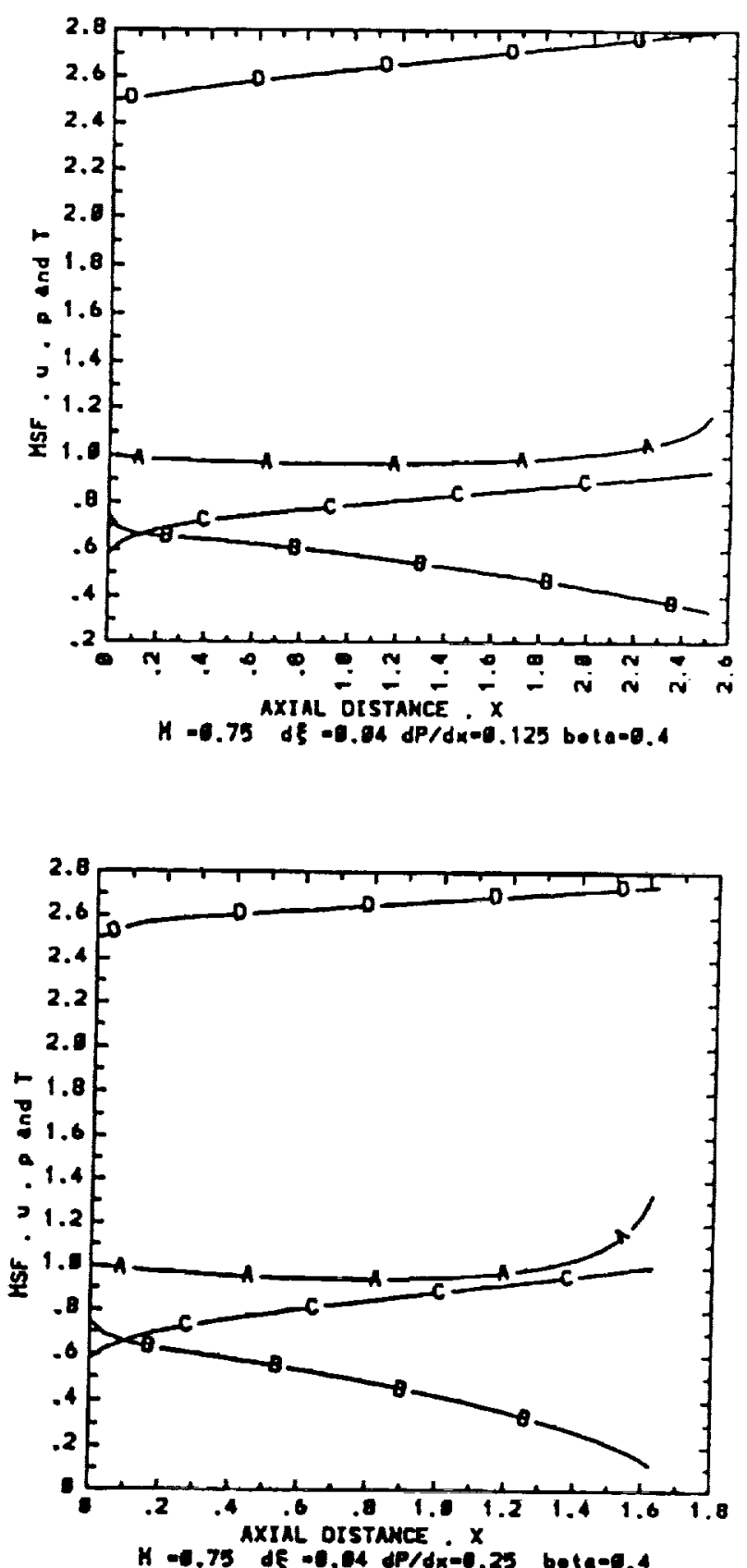

$N-4.75$ dE - 0.44 dP/dx-0.25 bete-a.4

$$
\mathrm{A}=\mathrm{MSF}, \mathrm{B}=\mathrm{u}_{\mathrm{a}}, \mathrm{C}=\mathrm{p}_{\mathrm{a}}, \mathrm{D}=\mathrm{T}_{\mathrm{a}}
$$

Fig. 1. Slender quasi-axisymmetric flow solutions for the effect of Mach number, external axial pressure gradient and swirl ratio. 
dure consists of two parts. In the first part a compatible set of initial profiles are obtained at $\xi=\xi$, and in the second part we use eqs. (4)-(8) and the compatibility equation to obtain $p, u, w$, $\rho, T$ and $V$ (or $v$ ).

\section{Numerical examples}

In the present numerical examples, the outer edge of the vortex, $\eta_{e}$, is taken as 10 , and 1000 grid points are used and hence $\Delta \eta_{\mathrm{e}}=0.01$. The
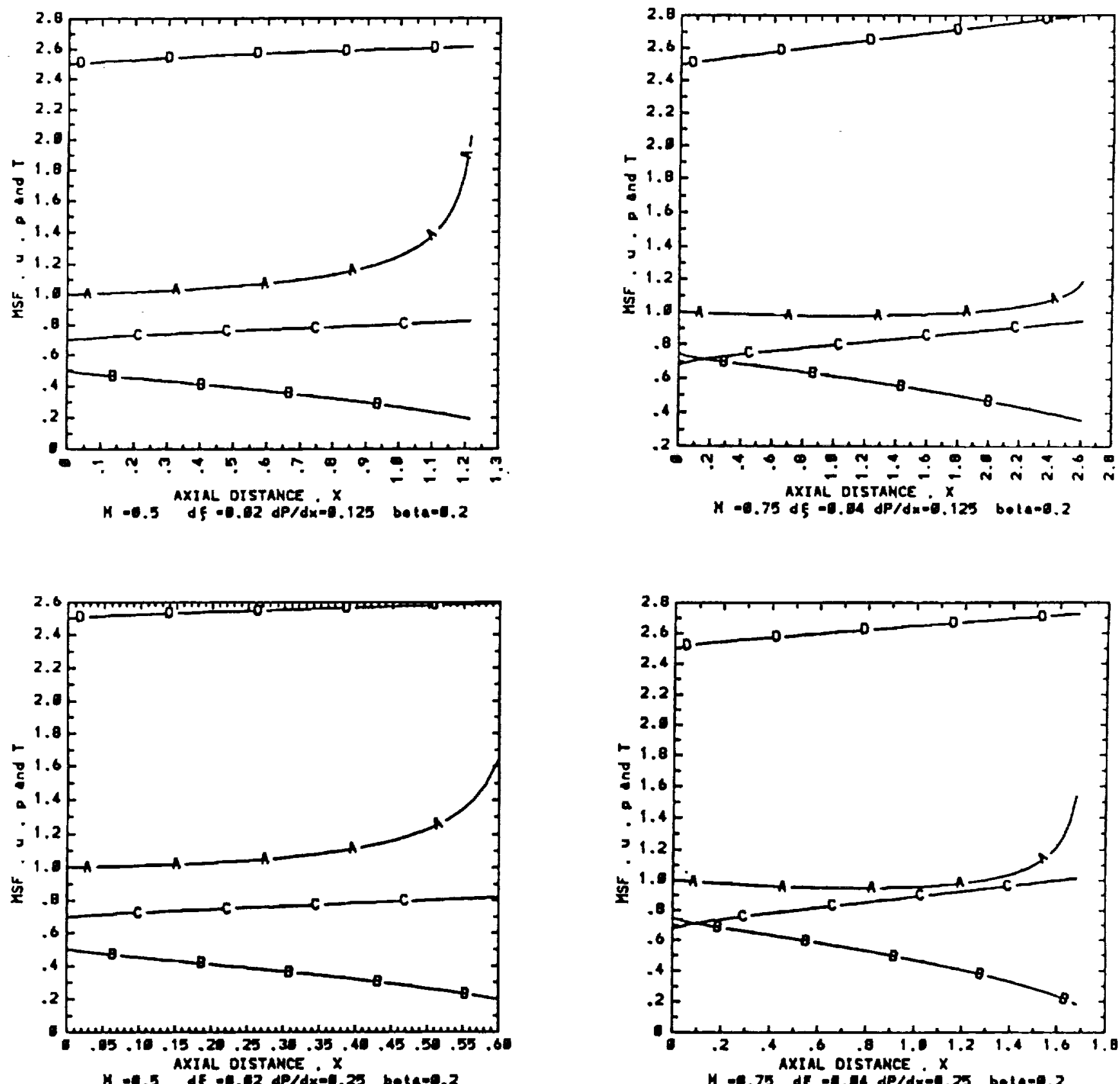

Fig. 1 (continued). 
results are shown for two Mach numbers: $M=0.5$ and 0.75 . The step size in the axial direction is 0.02 for $M=0.5$ and 0.04 for $M=0.75$. For each Mach-number case, we solve for two external axial pressure gradients; $(\partial p / \partial x)_{\mathrm{e}}=0.125$ and 0.25 and two swirl ratios: $\beta=(w / u)_{r=1}=0.2$ and 0.4 . The initial profiles for $u_{i}, w_{i}$ and $T_{i}$ are $u_{i}=$ constant, $w_{i}=\beta u_{i} r\left(2-r^{2}\right)$ for $r \leq 1$ and $w_{i}=\beta u_{i} / r$ for $r \geq 1$ and $T_{\mathrm{i}}=2.5$, respectively. Fig. 1 shows MSF, $u_{\mathrm{a}}, p_{\mathrm{a}}$ and $T_{\mathrm{a}}$ which are referred to by curves $\mathrm{A}$. $B, C$ and $D$; respectively. The results show that the breakdown length is more than doubled when the Mach number increases from 0.5 to 0.75 . They also show that while the outer boundary continuously increases for $M=0.5$, it initially decreases and then increases for $M=0.75$; see the A curves.
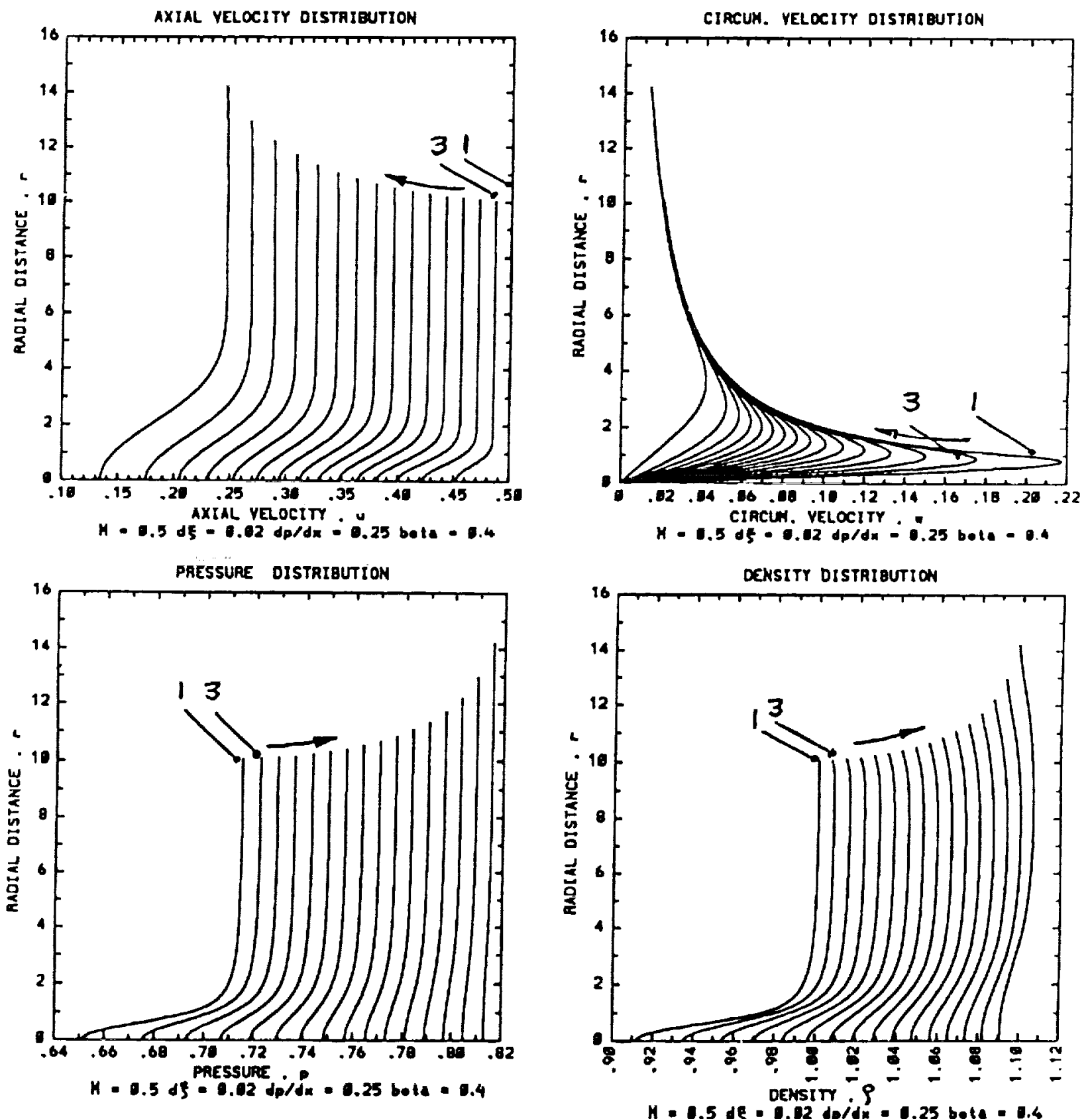

Fig. 2. Flow profiles for slender quasi-axisymmetric flows at $M=0.5$ and $0.75, \beta=0.4,(\mathrm{~d} p / \mathrm{d} x)_{e}=0.25$. 
The adverse pressure gradient at the vortex axis decreases faster for $M=0.75$ than for $M=0.5$. The results also show that the external axial pressure gradient is a dominant parameter on the breakdown length. As the external axial pressure gradient is doubled, the breakdown length substantially decreases. Doubling the swirl ratio slightly decreases the breakdown length.
Fig. 2 shows the profiles of $u, w, p$ and $\rho$ across $r$ at axial stations until the breakdown location for $M=0.5$ and 0.75 for the cases of $(\mathrm{d} p / \mathrm{d} x)_{\mathrm{e}}=0.25$ and $\beta=0.4$. The initial profiles are indicated by the number 1 and the next shown station is indicated by 3 . At $M=0.75$, it is noticed that the pressure and density gradients in the axial direction decrease faster than those at $M=0.5$.
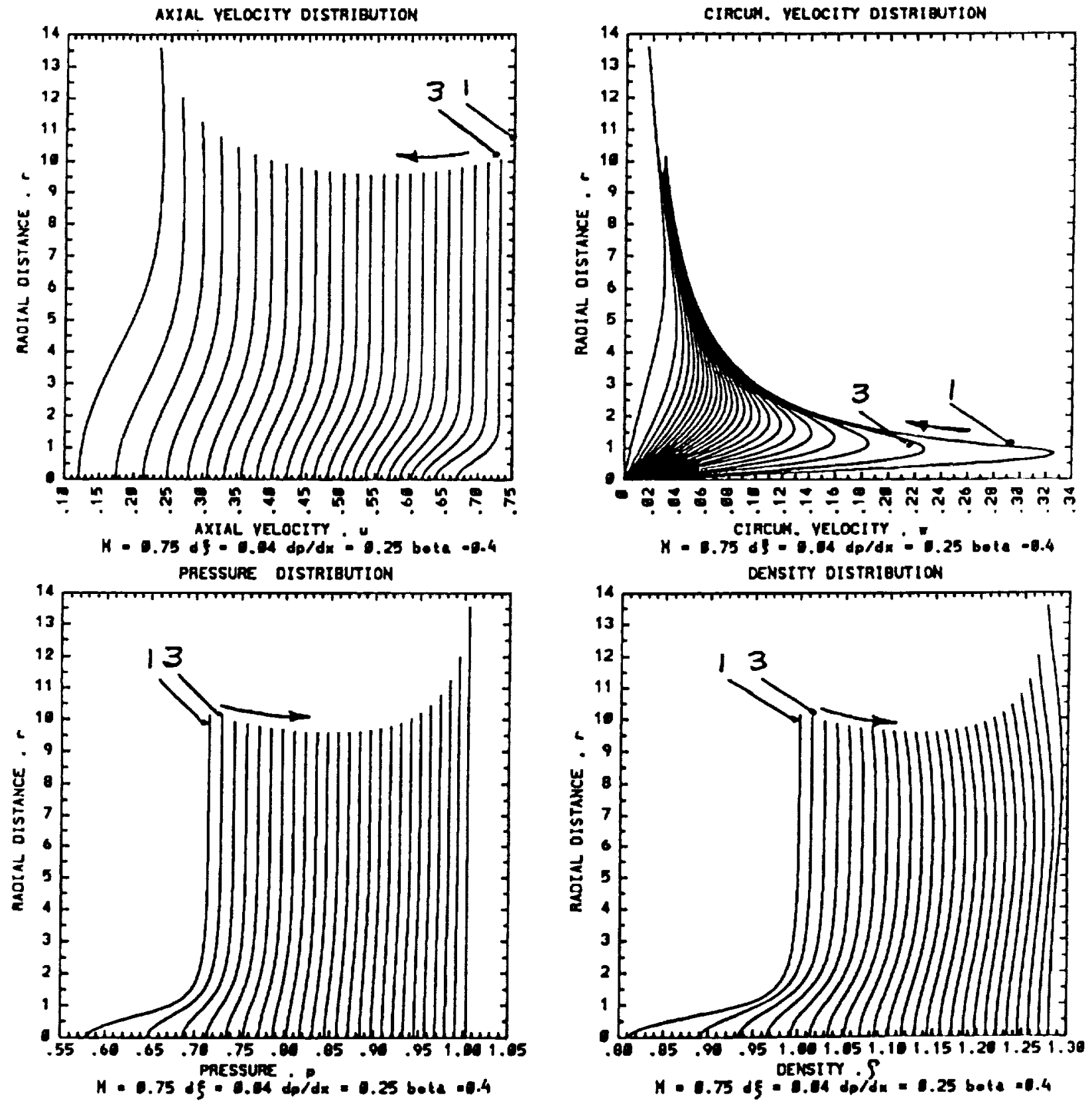

Fig. 2 (continued). 
The profiles show that the viscous diffusion at $M=0.75$ is larger than that at $M=0.5$.

Fig. 3 shows the profiles of $u, w, v$ and $p$ which has been computed by the present method and by an upwind Navier-Stokes solver for the case of $M=0.5, \beta=0.6$ and $(\mathrm{d} p / \mathrm{d} x)_{\mathrm{e}}=0$. For the Navier-Stokes solver a rectangular grid of $100 \times 51 \times 51$ in the axial direction and cross-flow
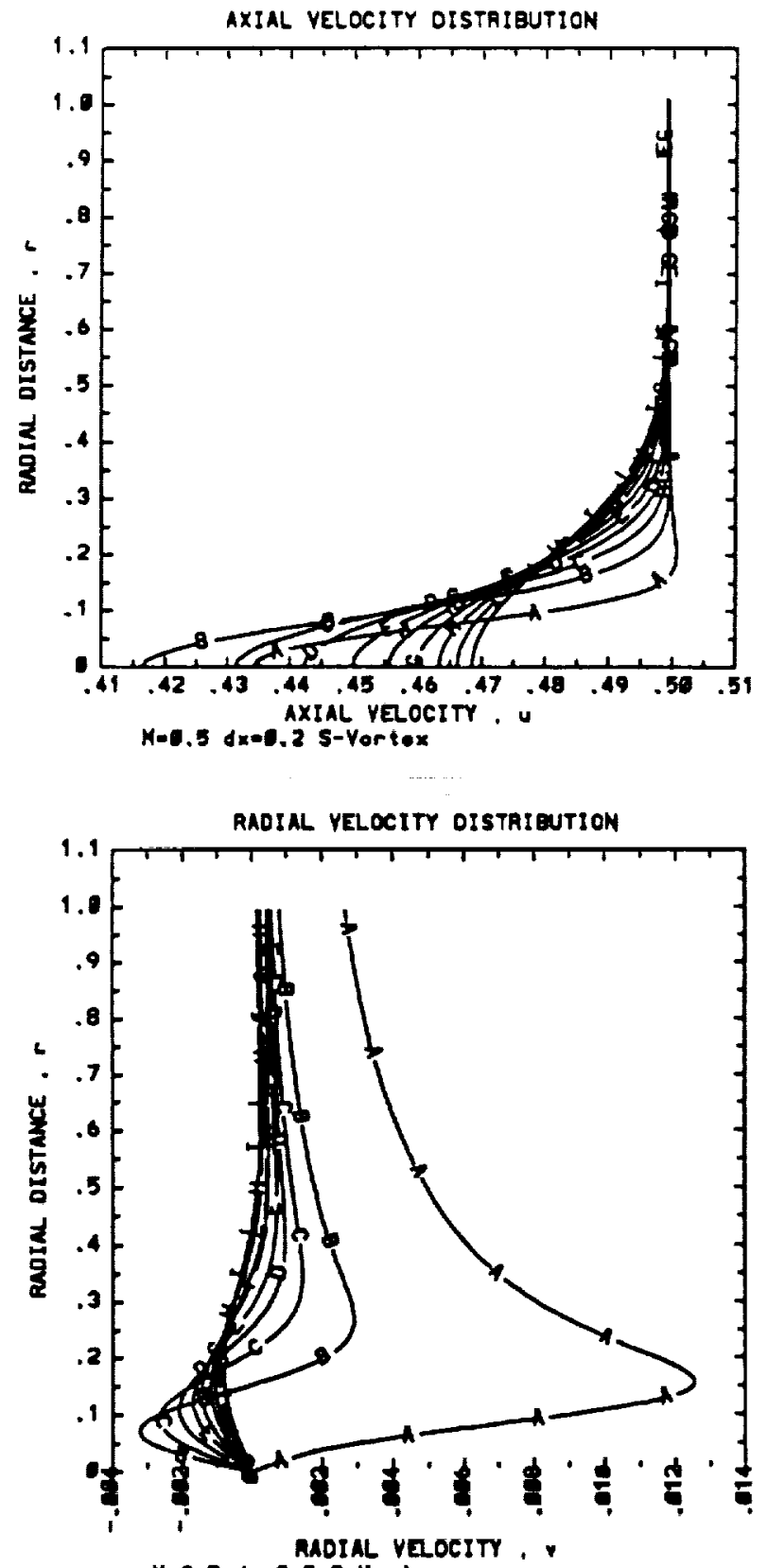

plane is used. The curves are labeled by the capital letters A, B, ... etc. Comparing the curves of the two sets, a remarkable agreement is seen.

It is concluded from the given numerical examples that increasing the flow Mach number has a favorable effect on the vortex breakdown location. The external axial pressure gradient is a dominant parameter on the vortex breakdown. Its effect
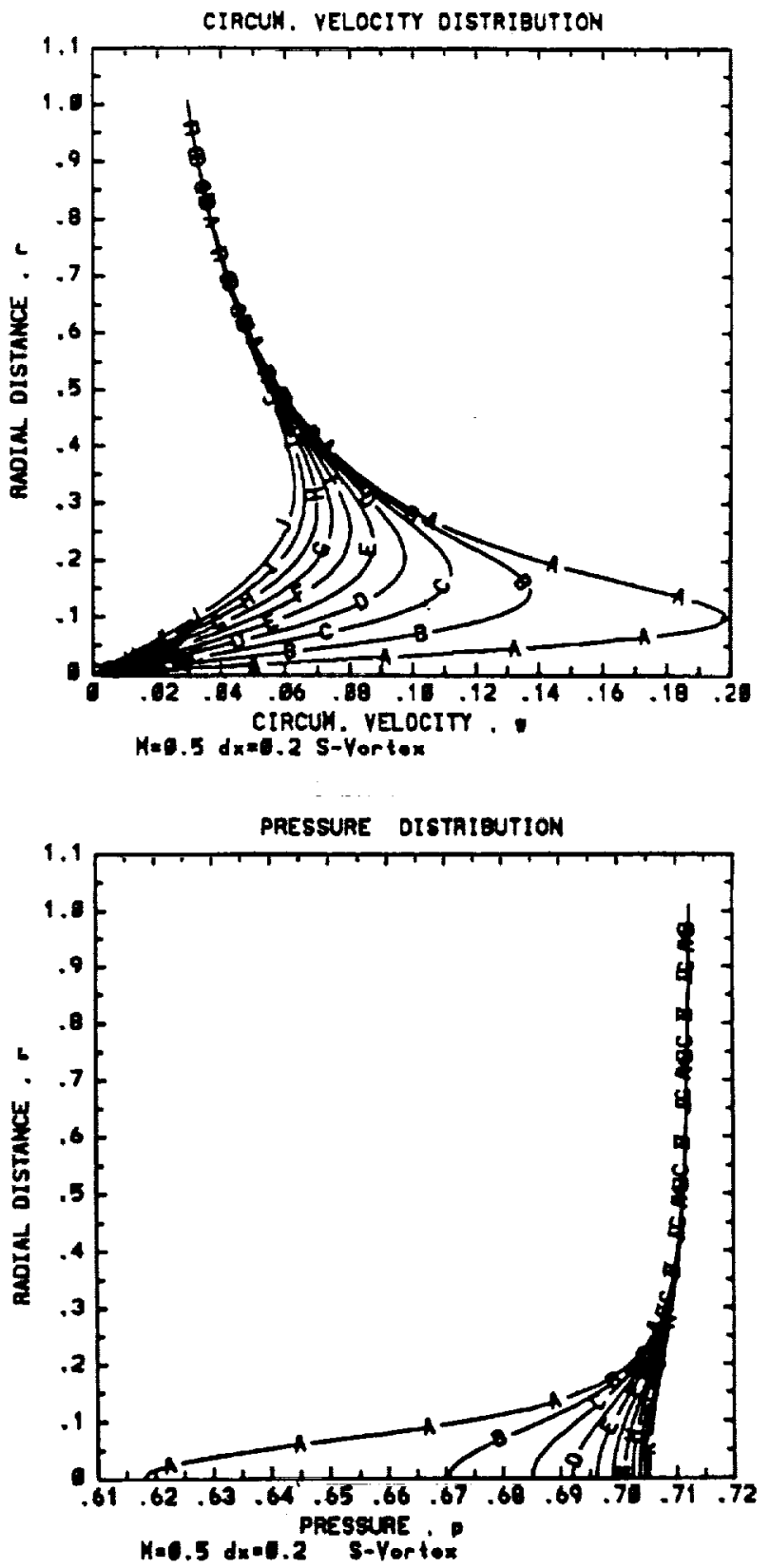

Fig. 3. Flow profiles for slender quasi-axisymmetric flows using the present method and the full Navier-Stokes equations, $M=0.5$, $\beta=0.6,(\mathrm{~d} p / \mathrm{d} x)_{\mathrm{e}}=0.0$. 

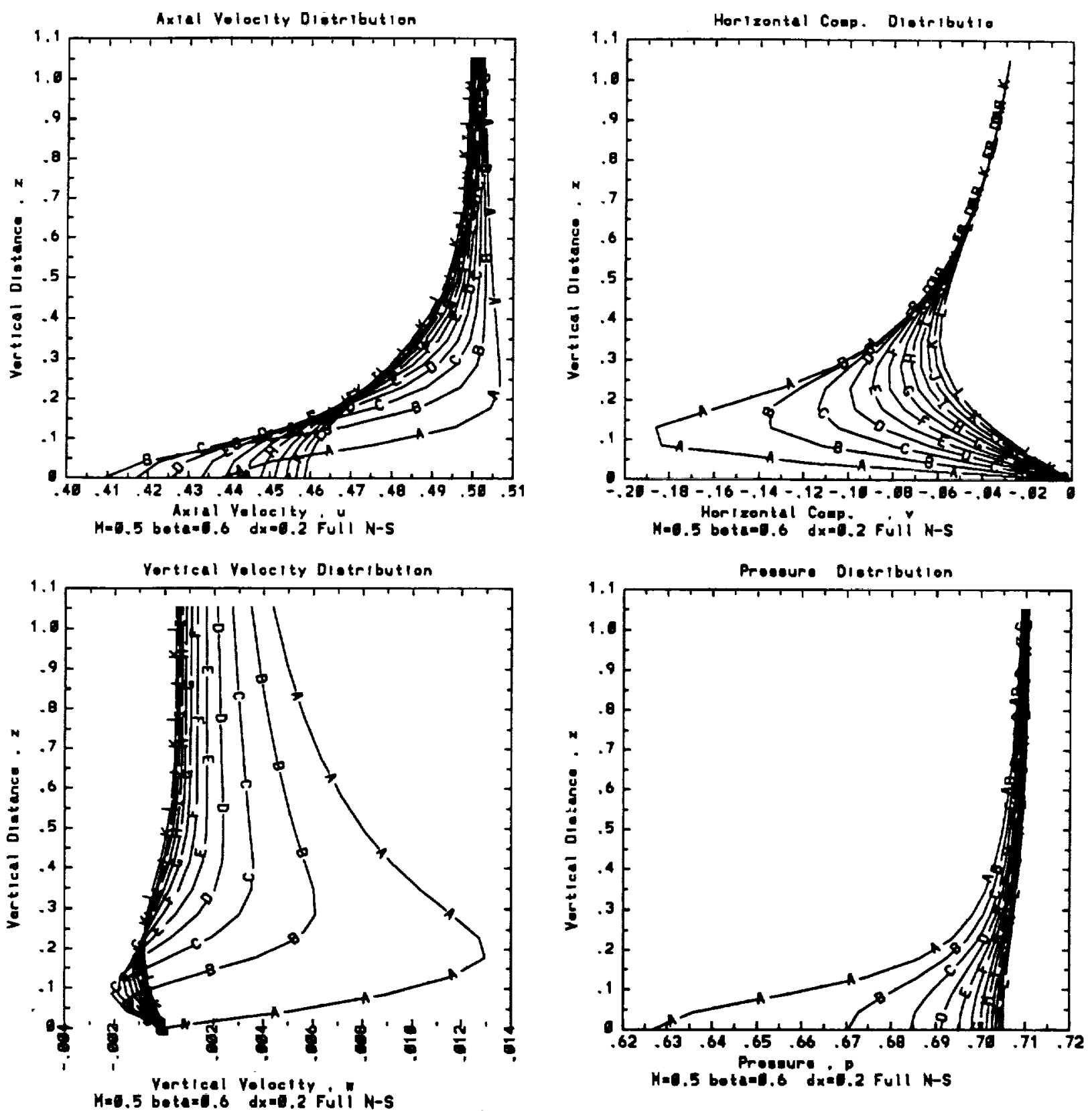

Fig. 3 (continued).

decreases as the Mach number is increased. Comparison of the present results with the full NavierStokes results gives a strong confidence in the present analysis. The present formulation and results are used to generate compatible initial profiles for the full Navier-Stokes solutions, and to provide data for breakdown-potential cases for accurate computations using the full NavierStokes equations. The full Navier-Stokes equa- tions are currently applied to these cases, so that we can solve for the flow in the breakdown region.

\section{Acknowledgement}

This research work is supported by the NASA Langley Research Center under Grant No. NAG$1-994$. 


\section{References}

[1] N.C. Lamboume and D.W. Bryer, Bursting of leading-edge vortices: Some observations and discussion of the phenomenon. Aeronautical Research Council, R\&M 3282 (1961).

[2] T. Sarpkaya, Vortex breakdown in swirling conical flows, AIAA J. 9 (1971) 1791 .

[3] S. Leibovich, Vortex stability and breakdown survey and extension, AlAA J. 23 (1984) 1194.

[4] M.P. Escudier and N. Zender, Vortex flow regimes, J. Fluid Mech. 115 (1982) 105.

[5] W.J. Grabowski and S.A. Berger, Solutions of the Navier-Stokes equations for vortex breakdown, J. Fluid Mech. 75 (1976) 525.
[6] M. Hafez, G. Kuruvila and M.D. Salas, Numerical study of vortex breakdown, J. Appl. Num. 2 (1987) 291.

[7] R.E. Spall, T. Gatski and C.E. Grosch, A criterion for vortex breakdown, ICASE Report $87-3$ (January 1987).

[8] J. Delery, E. Horowitz, O. Leuchter and J.L. Solignac, Fundamental studies of vortex flows. Rech. Aerosp. No. (1984) 1 .

[9] O. Metwally, G. Settles and C. Horstman, An experimental study of shock wave/vortex interaction, AIAA Paper 89.0082 (January 1989).

[10] C.H. Liu, E. Krause and S. Menne, Admissible upstream conditions for slender compressible vortices. AIAA Paper 86-1093 (July 1986).

[11] G. Copening and J. Anderson, Numerical solutions to three-dimensional shock/vortex interaction at hypersonic speeds, AIAA Paper 89-0674 (January 1989). 


\section{AIAA 91-0752}

COMPUTATION OF STEADY AND UNSTEADY COMPRESSIBLE QUASI-AXISYMMETRIC VORTEX FLOW AND BREAKDOWN

O. A. KANDIL AND H. A. KANDIL Old Dominion University, Norfolk, VA

C. H. LIU

NASA Langley Research Center, Hampton, VA

29th Aerospace Sciences Meeting January 7-10, 1991/Reno, Nevada

- For permission to copy or republish, contact the American Institute of Aeronautics and Astronautics 370 L'Entant Promenade, S.W., Washington, D.C. 20024 


\title{
COMPUTATION OF STEADY AND UNSTEADY COMPRESSIBLE QUASI-AXISYMMETRIC VORTEX FLOW AND́ BREAKDOWN
}

\author{
Osama A. Kandil* and Hamdy A. Kandil** \\ Old Dominion University, Norfolk, VA \\ C. H. Liu*** \\ NASA Langley Research Center, Hampton, VA
}

\begin{abstract}
The unsteady, compressible Navier-Stokes equations are used to compute and analyze compressible quasiaxisymmetric isolated vortices. The Navier-Stokes equations are solved using an implicit, upwind, flux-difference splitting finite-volume scheme. The developed threedimensional solver has been verified by comparing its solution profiles with those of a slender, quasi-axisymmetric vortex solver for a subsonic, isolated quasi-axisymmetric vortex in an unbounded domain. The Navier-Stokes solver is then used to solve for a supersonic quasiaxisymmetric vortex flow in a configured circular duct. Steady and unsteady vortex-shock interactions and breakdown have been capured. The problem has also been calculated using the Euler solver of the same code and the results are compared with those of the Navier-Stokes solver. The effect of the initial swirl has been tentatively studied.
\end{abstract}

\section{Introduction}

The phenomenon of vortex breakdown or bursting was observed in the water vapor condensation trails along the leading-edge vortex cores of a gothic wing. Two forms of the leading-edge vortex breakdown, a bubble type and a spiral type, have been documented experimentally'. The bubble type of vortex breakdown shows an almost axisymmerric sudden swelling of the core into a bubble, and the spiral type of vortex breakdown shows an asymmetric spiral filament followed by a rapidly spreading turbulent flow. Both types are characterized by an axial stagnation point and a limited region of reversed axial flow. Much of our knowledge of vortex breakdown has been obtained from experimental studies of pipe flows where both types of breakdown and other types as well were generated and documented ${ }^{2-4}$. The major effort of numerical simulation of vortex breakdown flows has been focused on incompressible, quasi-axisymmetric isolated vortices. Grabowsti and Berges used the incompressible, quasiaxisymmetric Navier-Stokes equations to study isolated vortex flow in an unbounded region. Hafez, et. a ${ }^{6}$ solved the incompressible, steady, quasi-axisymmetric Euler and

\footnotetext{
-Profescor ad Eminens Scholer. Depertemene of Mecharical Engineartas and Mechnice, Arsocise Fellow, ALA

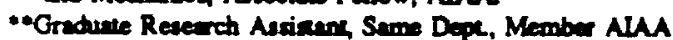

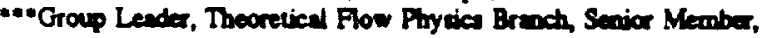
NAA
}

This paper is declared a work of the U.S. Government and is not subject to copyright protection in the United States.
Navier-Stokes equations using the stream function. vorticity formulation for isolated vortex flows. They predicted vortex breakdown flows similar to those of Garbowski and Berger. Menne ${ }^{7}$ has also used the stream function vorticity formulation for unsteady, incompress. ible quasi-axisymmetric isolated vortex flow. Menne and $\mathrm{Liu}^{8}$ used the Navier-Stokes equations to study threedimensional incompressible flows in a tube. Spall, et. al ${ }^{9}$, presented a study of the structure and dynamics of bubbletype vortex breakdown in incompressible flows using the vorticity-velocity formulation. For more information on the physical and computational aspects of the incompressible vortex breakdown, the reader can refer to the paper by Krause ${ }^{10}$.

Flows around highly swept wings and slender wingbody configurations at transonic and supersonic speeds and at moderate to high angles of attack are characterized by vortical regions and shock waves, which interact with each other. Other applications which encounter vortex-shock interaction include a supersonic inlet ingesting a vortex and injection into a supersonic combustor to enhance the mixing process, see Delery, et. al ${ }^{11}$ and Metwally, seules, and Horsuman ${ }^{12}$. Figure 1 shows these examples, where Fig. 1.a and 1.b are taken from ref. 11 and Fig.1.c is taken from ref. 12. These problems and others call for developing computational schemes to predict, study and control compressible vortex flows and their interaction with shock waves. Unfortunately, the literature lacks this type of analysis with the exception of the preliminary work of Liu, Krause and Menne ${ }^{13}$, Copening and Anderson ${ }^{14}$, Delery, et. al ${ }^{11}$, Kandil and Kandil ${ }^{15}$, Meadows, Kumar and Hussaini ${ }^{16}$.

In this paper, we use the unsteady, compressible full Navier-Stokes equations to compute and analyze compressible and supersonic quasi-axisymmetric isolated vortices. An implicit, upwind, flux-difference spliting finitevolume scheme, which is based on the Roe scheme, has been used to solve the full Navier-Stokes equations. The three-dimensional solver, which is called "FTNS3D", has been used to solve two problems of isolated vortex flows. The first problem is that of a subsonic, isolated quasiaxisymmetric vortex in an unbounded domain. This case has been verified by comparing the flow-profiles solutions with those of the slender vortex solver of ref. 15. Next, the three-dimensional Navier-Stokes equations are used to solve for a supersonic quasi-axisymmetric vortex flow 
in a configured circular duct ${ }^{11}$. Since the flow is quasiaxisymmetric, the solution is obtained by forcing the flow variables to be equal on two axial (meridian) planes. Solutions for steady and unsteady vortex-shock interactions and breakdown have been obtained. These solutions are compared with those of the Euler equations using the inviscid version of same solver. Effects of the initial swirl ratio has been tentatively investigated.

\section{Formulation}

The conservative form of the dimensionless, unsteady, compressible, full Navier-Stokes equations in terms of time-independent, body-conformed coordinates $\xi^{l}, \xi^{2}$ and $\xi^{3}$ is given by

$$
\frac{\partial \tilde{Q}}{\partial t}+\frac{\partial \bar{E}_{m}}{\partial \xi^{m}}-\frac{\partial\left(\bar{E}_{v}\right)_{s}}{\partial \xi^{\natural}}=0 ; m=1-3, s=1-3
$$

where

$$
\begin{gathered}
\xi^{\mathrm{m}}=\xi^{\mathrm{m}}\left(\mathrm{x}_{1}, \mathrm{x}_{2}, \mathrm{x}_{3}\right) \\
\overline{\mathrm{Q}}=\frac{\hat{\mathrm{q}}}{\mathrm{J}}=\frac{1}{\mathrm{~J}}\left[\rho, \rho \mathrm{u}_{1}, \rho \mathrm{u}_{2}, \rho \mathrm{u}_{3}, \rho \mathrm{e}\right]^{\mathrm{t}}
\end{gathered}
$$

$$
\begin{aligned}
\tilde{E}_{\mathrm{m}} & \equiv \text { inviscid flux } \\
& =\frac{1}{\mathrm{~J}}\left[\partial_{\mathrm{k}} \xi^{\mathrm{m}} \hat{\mathrm{E}}_{\mathbf{k}}\right]^{\mathrm{t}} \\
& =\frac{1}{\mathrm{~J}}\left[\rho \mathrm{U}_{\mathrm{m}}, \rho \mathrm{u}_{1} \mathrm{U}_{\mathrm{m}}+\partial_{1} \xi^{\mathrm{m}} \mathrm{p}, \rho \mathrm{u}_{2} \mathrm{U}_{\mathbf{m}}\right. \\
& \left.+\partial_{2} \xi^{\mathrm{m}} \mathrm{p}, \rho \mathrm{u}_{3} \mathrm{U}_{\mathrm{m}}+\partial_{3} \xi^{\mathrm{m}} \mathrm{p},(\rho \mathrm{e}+\mathrm{p}) \mathrm{U}_{\mathrm{m}}\right]^{\mathrm{t}}
\end{aligned}
$$

$$
\begin{aligned}
& \left(\bar{E}_{v}\right), \equiv \text { viscous and heat-conduction flux in } \xi^{*} \\
& =\frac{1}{\mathrm{~J}}\left[0, \partial_{k} \xi^{*} n_{\mathrm{k}}, \partial_{\mathrm{k}} \xi^{*} n_{\mathrm{k}}, \partial_{\mathrm{k}} \xi^{*} n_{\mathrm{k}}\right. \text {, } \\
& \left.\partial_{k} \xi^{3}\left(u_{n} \eta_{k n}-q_{k}\right)\right]^{3} ; \quad k=1-3, n=1-3 \\
& \mathrm{U}_{\mathrm{m}}=\partial_{\mathrm{k}} \xi^{\mathbf{m}} \mathrm{u}_{\mathrm{k}}
\end{aligned}
$$

The first element of the three momentum elements of Eq. (5) is given by

$$
\begin{aligned}
\partial_{k} \xi^{*} n_{1} & \equiv \frac{M_{x} \mu}{R e}\left[\left(\partial_{k} \xi^{2} \partial_{1} \xi^{ \pm}-\frac{2}{3} \partial_{1} \xi^{a} \partial_{k} \xi^{ \pm}\right) \frac{\partial u_{k}}{\partial \xi^{2}}\right. \\
& \left.+\partial_{k} \xi^{2} \partial_{k} \xi^{2} \frac{\partial u_{1}}{\partial \xi^{2}}\right]
\end{aligned}
$$

The second and third elements of the momentum elements are obtained by replacing the subscript 1 , everywhere in
Eq. (7), with 2 and 3, respectively. The last element of Eq. (5) is given by

$$
\begin{aligned}
\partial_{k} \xi^{3}\left(u_{p} r_{p p}\right. & \left.-q_{k}\right) \equiv \frac{M_{x} \mu}{R e}\left[\left(\partial_{k} \xi^{s} \partial_{p} \xi^{n}\right.\right. \\
& \left.-\frac{2}{3} \partial_{p} \xi^{s} \partial_{k} \xi^{n}\right) u_{p} \frac{\partial u_{k}}{\partial \xi^{n}} \\
& +\partial_{k} \xi^{s} \partial_{k} \xi^{n} u_{p} \frac{\partial u_{p}}{\partial \xi^{n}} \\
& \left.+\frac{1}{(\gamma-1) P_{r}} \partial_{k} \xi^{*} \frac{\partial\left(a^{2}\right)}{\partial \xi^{n}}\right] ; p=1-3
\end{aligned}
$$

The reference parameters for the dimensionless form of the equations are $L, a_{x}, L / a_{x}, \rho_{x}$ and $\mu_{x}$ for the length, velocity, time, density and molecular viscosity, respectively. The Reynolds number is defined as $\mathrm{Re}=$ $\rho_{\infty} V_{\infty} L / \mu_{\infty}$, where $L$ is the initial radius of the vortex or the duct inlet radius. The pressure, $p$, is related to the total energy per unit mass and density by the gas equation

$$
p=(\gamma-1) \rho\left[e-\frac{1}{2}\left(u_{1}^{2}+u_{2}^{2}+u_{3}^{2}\right)\right]
$$

The viscosity is calculated from the Sutherland law

$$
\mu=\mathrm{T}^{3 / 2}\left(\frac{1+\mathrm{C}}{\mathrm{T}+\mathrm{C}}\right), \mathrm{C}=0.4317
$$

and the Prandu number $P_{r}=0.72$. In Eqs. (1)-(8), the indicial notation is used for convenience.

\section{Computational Scheme}

The computational scheme used to solve the full Navier-Stokes equations is an implicit, upwind, fluxdifference spliting, finite-volume scheme. It employs the flux-difference spliting scheme of Roe. The Jaco. bian matrices of the inviscid fluxes, $A_{1}=\frac{\partial E_{a}}{\partial q} ; s=1-3$, are split into backward and forward fluxes according to the signs of the eigenvalues of the inviscid Jacobian matrices. Flux limiters are used to eliminate oscillations in the shock region. The viscous and heat-flux terms are centrally differenced. The resulting difference equation is solved using approximate factorization in the $\xi^{1}, \xi^{2}$ and $\xi^{3}$ directions. In addition to the three-dimensional flows, the present computer program can solve for axisymmetric and quasi-axisymmetric flows. The resulting computer program can also be used to solve the Euler equations. This code is a modified version of the CFL 3D which is currently called "FTNS3D". The modifications have been developed by the present authors. 


\section{Computational Applications}

In this section, two computational applications are presented. The first application is that of a steady, subsonic quasi-axisymmetric vortex flow in an unbounded domain. The purpose of this application is to verify the Navier-Stokes solver by comparing the results of this case with those of a previously developed slender vortex solver, see ref. 15 . The second application is that of a steady and unsteady, supersonic quasi-asymmetric vortex flow in a configured circular duct. This application is solved by using pseudo-time stepping and accurate-time stepping. The results are compared with those of the Euler equations solver of the same computer program. Next, we consider each application and discuss its results.

Steady Subsonic Quasi-Axisymmetric Vortex Flow in an Unbounded Domain

Here, the three-dimensional Navier-Stokes is used to solve for an isolated quasi-axisymmetric flow. The computational domain for the Navier-Stokes equations is a parallelopiped rectangular domain with a square crosssection of $10 \times 10$ units. The downstream length is 10 units. The rectangular grid consists of $51 \times 51 \times 100$ points in the two directions of the square section and in the axial direction, respectively. The grid is clustered algebraically at the axis of the parallelopiped domain. The step size in the axial direction is 0.1 .

For the slender-vortex solver ${ }^{15}$, the computational domain is a cylindrical one and the solution is obtained on one meridian plane having a radius of 10 and a length of 10 . The number of grid points in the radial direction is 1000 . The step size in the axial direction is 0.1 . The initial profile for the slender vortex solver are given by $\mathrm{u}_{\mathrm{i}} \equiv$ axial velocity $=$ constant, $w_{\mathrm{i}} \equiv$ tangential velocity $=$ $\beta u_{i} r\left(2-r^{2}\right)$ for $r \leq 1$ and $w_{i}=\beta u_{i} / r$ for $r \geq 1$ and $T_{i} \equiv$ temperature $=2.5$, where $\beta=0.6$. The Mach number at the outer radius of the initial station, $M_{0}=0.5$. The other compatible initial profiles for $p_{i}, v_{i}$ and $\rho_{i}$ (pressure, radial velocity and density; respectively) are obtained from the radial momentum equation, a compatibility equation ${ }^{13}$ and the equation of state. The external axial pressure gradient is selected as $\left(\frac{\partial p}{\partial x}\right)=0$. The extemal boundary conditions on the cylindrical outer boundary are obtained by using the Euler equations 10 match the outer profiles to those of the viscous core in order to obtain the conditions on $u_{6}, w_{0}, T_{\text {, }}$ and $\rho_{0}$.

For the Navier-Stokes solver, the initial profiles are obtained from the previous initial profiles by interpolating the slender vortex profiles on the rectangular grid at the initial station. The Reynolds number of the Navier-Stokes solver for this case is set at 100 .

Figure 2 shows the Navier-Stokes solutions on the left and the stender-vartex solutions on the right. The figure shows comparison of the profiles of axial velocity $u$, tangential velocity $w$, radial velocity $v$, pressure $p$ and density $\rho$ at the same axial stations which are marked by $A, B, C \ldots$. . It is remarkable to see the excellent agreement between the Navier-Stokes solutions and the slender-vortex solutions at every axial station. It should be emphasized here that the Navier-Stokes solutions for this quasi-axisymmetric flow have been obtained by using the three-dimensional solver on a three-dimensional grid.

Having verified the Navier-Stokes solver, the next problem to consider is the supersonic vortex flow in a configured circular duct.

\section{Supersonic Quasi-Axisymmetric Vortex in} a Configured Circular Duct

Figure 3 shows a configured circular duct which consists of a straight cylindrical par at the inlet that is followed by a shor, diverging cylindrical par. At $\mathrm{x}=$ 0.75 and beyond, the duct radius is kept constant and a convergent-divergent nozzle with a throat radius of 0.95 is attached. The overall dimensions of the duct is $1 \times 2.90$. This configured duct ensures that the inlet supersonic flow will becomes supersonic at the exit. Moreover, the convergent part near the inlet ensures the stability of the formed shock in the inlet region. This configured duct has also been used by Delery, et. al " for their Euler equations computations in an attempt to computationally model an experimental set up. It should be pointed here that the Euler equations, used by Delery, et. al, assume isenthalpic flow in order to drop the energy equation. This is a serious approximation since the upstream fow is a rotational flow. Moreover, as our present calculations show, the flow is actually unsteady and hence, the isenthalpic assumption is not valid.

The Navier-Stokes solver is used to compute this flow case by using a grid of $200 \times 51$ on two meridian planes, where the 200 points are in the axial direction and the 51 points are in the radial direction. The grid is clustered at the center line (CL) and at the wall. It is also clustered in the diverging part near the inlet. The two meridian planes are spaced circumferentially at a certain angle so that the aspect ratio of the minimum grid size will be less than 2. The upstream Mach number is $M_{x}=1.75$ and the Reynolds number for the Navier-Stokes computations is $10^{4}$. The initial profile for the tangential velocity is given by

$$
\frac{w}{U_{\infty}}=\frac{k_{\infty}}{r}\left[1-\exp \left(-\frac{r^{2}}{r_{m}^{2}}\right)\right]
$$

where $U_{\infty}=1.74, r_{m}=0.2$ and $k_{0}=0.1$. The maximum $\pi$ is at $r=0.224$ and it is equal to 0.32 . The radial velocity, $v$, at the initial station is set equal to zero and the radial momentum equation is integrated to obtain the initial pressure profile. Finally, the density $\rho$ is obtained from the definition of the speed of sound for the inlet flow. With these compatible set of profiles, the computations for both the Navier-Stokes equations and the Euler equations star. The exit boundary conditions are obtained by extrapolation from the interior since the flow is supersonic at the exit. The wall boundary conditions follow the 
lypical Navier-Stokes and Euler equations solid-boundary conditions. These computations have been carried out on the CRAY YMP of the NAS-Ames computational facilities. The CPU time is $30 \mu \mathrm{s} /$ grid point/iteration for the Navier-Stokes calculation and $20 \mu \mathrm{s} / \mathrm{grid}$ point/iteration for the Euler equations.

\section{a. Pseudo-Time-Stepping Solutions}

Figure 4 shows the pseudo-time stepping solutions of the Navier-Stokes (NS) equations on the left and the Euler (E) equations on the right. Each column in the figure shows How properties at the center line, the total Mach contours, the streamlines throughout the duct and a blow up of the streamlines in the vortex breakdown region.

The figure of properties along the center line of the NS solution shows a strong shock at the inlet. Behind the shock, the pressure and density sharply increase and the axial velocity decreases to a negative value (upstream flow) at $x=0.10$. The axial velocity becomes more negative one more time at $x=0.4$ indicating the formation of another bubble. At $x=0.7$, the axial velocity becomes positive and it continuously increases ill the duct exit. The Mach number contours of the NS solutions shows the shock system near the inlet. The shock at the center line is a normal strong shock, then it becomes an oblique strong shock, again it becomes a normal strong shock, and at the wall it becomes an oblique weak shock (supersonicsupersonic flows upstream and downstream of the shock). It is seen that the oblique shock at the wall is followed by a separation bubble (see the streamline figure) which is due to the shock and the divergence of the duct at this location. The streamlines figure of the NS solution shows a very large vortex-breakdown bubble and the blow-up figure of the streamlines shows another small bubble upstream of the large one.

The figure of Euler solution shows similar vortex breakdown features with a few differences from the NS solution. These differences are due to the absence of viscous forces. The figure of properties along the center line of the E solution shows a strong shock at the inlet. Behind the shock, the pressure and density sharply increase to a level higher than that of the NS solution. The axial velocity decreases to a negative value which is less than that of the NS solution. The Mach-number contours of the $E$ solution show the shock system near the inlet. The shock at the center line is a normal strong system, then it becomes an oblique weak shock and at the wall it becomes a strong normal shock where there is no shock induced separation. Another normal shock develops at $x=0.61$, where the axial velocity becomes substantially negative. The streamline figure shows three vortex-breakdown bubbles; two small counter rotating bubbles and a third large bubble. The size of vortex-breakdown bubbles of the $E$ solutions is larger than those of the NS solution.

It should be stressed here that this is the first time, that we know of, such solutions have been presented for supersonic vortex breakdown.

\section{b. Time-Accurate-Stepping Solutions}

It has been noticed during the pseudo-time stepping solutions that the residual-enor dropped two orders of magnitude and then it went through oscillations. It is then decided that time-accurate-stepping solutions must be checked. The same problem was recalculated using the $N S$ equations and $E$ equations with $\Delta t=0.005$. Figures 5-8 show snap shots of the time accurate solutions of the NS equations on the left and the E equations on the right. The snap shots are shown every 400 ime steps. The figures show the streamlines (Fig. 5), blow-up of streamlines in the breakdown region (Fig. 6), total Machnumber contours (Fig. 7 ) and flow properties at the center line (Fig. 8).

Following the snap shots of NS streamlines (Figs. 5 and 6), we see a large bubble forming at the center line at the time $t=4$. At $t=6$, the bubble expands in the upstream and lateral directions. During this time period $t$ $=4-6$, the Mach contours (Fig. 7) show the shock system at the inlet moving in the upstream direction. At $t=8$, two bubbles appear and are convected in the downstream direction. The Mach contours show that the shock moves upstream and reaches the inlet as a normal surong shock. At $t=10$, a new vortex breakdown occurs producing new small bubbles which combine to form a large bubble at $t=12$. It should be noted here that the bubble at $t=12$ resembles that at $t=4$. This suggests that the vortex breakdown process is almost periodic. To show the periodicity of the breakdown, one has to pick up the exact corresponding snap shots which are one period apart. This search is underway and it will be shown in the near future. The solutions at $t=12,14$ and 16 show a trend of repetition of the breakdown process. It should be noted that the separation bubble at the wall goes through a periodic process of convection and reproduction. The Mach contours in the period of $t=10-16$ show that the shock system moves in the downstream direction again. Figure 8 shows snap shots of the corresponding properties variations along the center line. It shows the shock motion and the motion of the negative values of the axial velocity.

Following the snap shots of the E equations, we see that a large vortex appears at the center line at $t=4$. $A t=6$, the vortex grows up and extends laterally and upstreans. At $t=8$, it is convected downstreams and another vortex appears behind the shock near the inlet. Figure 7 shows that the shock system near the inlet moves upstreams in the period of $t=4-8$. The convection process and production of new vortices behind the shock continue thereafter (Figs. 5 and 6) while the shock system moves downstreams. It should be noted that the motion of the shock system of the $E$ solutions is larger than that of the NS solutions. The reason is the absence of the viscosity and hence the flow slips at the wall. Moreover, there is no separation bubble at the divergent part of the channel. The flow properties at the center line show the 
motion of the shock system and the motion of the negative values of the axial velocity.

\section{Effect of Increasing Swirl Ratio}

Next, the flow conditions and duct dimensions are kept constant while the initial swirl ratio is increased to $3=0.38$. The pseudo-time-stepping results are shown in Fig. 9. It is seen that the number of vortex breakdown bubbles increases to three instead of the two bubbles of the previous case, Fig. 4. Moreover, we notice that the shock system of the present case is nearer to the inlet in comparison with the shock system of the previous case. Figure 10 show snap shots of the time-accurate-stepping solutions of this case at $t=5,12.5$ and 15.5. Again, we see the vortex breakdown process of production and convection and the associated oscillation of shock system. The solution shows larger size and more number of bubbles in comparison with those of the previous case (Figs. 5-7). The time step of this case is the same as that of the previous one, $\Delta t=0.005$.

\section{Concluding Remarks}

The unsteady, compressible full Navier-Stokes equations are used to compute and analyze compressible and supersonic quasi-axisymmetric isolated vortices. First. the three-dimensional Navier-Stokes solver has been verified by solving for a subsonic, isolated quasiaxisymmetric vortex in an unbounded domain. The results have been compared with those of a slender-vortex solver and they are in excellent agreement. Second, the three-dimensional Navier-Stokes and Euler solvers are used to solve for a supersonic quasi-axisymmetric vortex in a configured circular duct. The duct is designed such that the inflow and outflow conditions are supersonic. The quasi-axisymmeric solution is obcained by forcing the flowfield vector to be equal on two meridian planes in close proximity of each ocher. For the first time, we have obtained supersonic vortex breakdown solutions behind a shock. The time-accurate solution of the problem shows that the vortex breakdown bubbles and the shock system ahead of them are time dependent. The solution strongly indicates that the vortex breakdown process and the motion of the shock system are periodic. The Euler solution shows larger size and more number of bubbles than those of the Navier-Stokes solution. The Euler solution also shows that the amplitude of the shock oscillation is larger than that of the Navier-Stokes solution. Increasing the initial swirl ratio shows that the size and number of vonex-breakdown bubbles increase. These results are vital for the mixing process in scramjets and their design for the best performance and efficiency. A very careful parametric study is underway to show the effects of the swirl ratio, Mach number, Reynolds number and relative dimensions of the duct Three-dimensional solutions are currently being developed.

\section{Acknowledgements}

This research work is suppored by the NASA Langley Research Center under Grant No. NAG-1-994. The authors would like also to acknowledge the computational time provided through a grant from the NAS-Ames com. putational facilities.

\section{References}

1. Lamboume, N. C. and Bryer, D. W., "Bursing of Leading-Edge Vortices: Some Observations and Discussion of the Phenomenon," Aeronautical Research Council, R\&M 3282, 1961.

2. Sarpkaya. T., "Vortex Breakdown in Swirling Conical Flows," AIAA Journal, Vol. 9, No. 9, Sept. 1971. pp. 1791-1799.

3. Leibovich, S., "Vortex Stability and Breakdown Survey and Extension," AIAA Joumal, Vol. 23, No. 9, Sept. 1984, pp. 1194-1206.

4. Escudier, M. P. and Zender, N., "Vortex Flow Regimes," Joumal of Fluid Mechanics, Vol. 115. 1982, pp. 105-122.

5. Grabowski, W. J. and Berger, S. A., "Solutions of the Navier-Stokes Equations for Vortex Breakdown," Joumal of Fluid Mechanics, Vol. 75, Part 3, 1976, pp. 525-544.

6. Hafez, M., Kuruvila, G. and Salas, M. D., "Numerical Sudy of Vortex Breakdown," Journal of Applied Numerical Mathematics, No. 2, 1987, pp. 291-302.

7. Menne, S., "Vortex Breakdown in an Axisymmetric Flow," AIAA 88-0506, January 1988.

8. Menne, S. and Liu, C. H., "Numerical Simulation of a Three-Dimensional Vortex Breakdown," Z. Flugwiss. Welturaumforsch.14, 1990, pp. 301-308.

9. Spall, R. E., Gatski, T. B. and Ash, R. L., "The Structure and Dynamics of Bubble-Type Vortex Breakdown," Proc. R. Soc., London, A429, 1990, pp. 613-637.

10. Krause, E., "Vortex Breakdown: Physical Issue and Computational Simulation," Third International Congress of Fluid Mechanics, Cairo, Egypt, January 1990. Vol. 1, pp. 335-344.

11. Delery, J., Horowitz, E., Leuchter, O. and Solignac, J. L., "Fundamental Studies of Vortex Flows," La Recherche Árospatiale, No. 1984-2, 1984, pp. 1-24.

12. Metwally, O., Settles, G. and Horstman, C., "An Experimental Study of Shock Wave/Vortex Interaction," AIAA 89-0082, January 1989.

13. Liu, C. H., Krause, E. and Menne, S., "Admissible Upstream Conditions for Slender Compressible Vortices," ALAA 86-1093, 1986. 
14. Copening, G. and Anderson, J., "Numerical Solutions to Three-Dimensional Shock/Vortex Interaction at $\mathrm{Hy}$ personic Speeds," AIAA 89-0674, January 1989.

15. Kandil, O. A. and Kandil, H. A., "Computation of Compressible Quasi-Axisymmetric Slender Vortex Flow and Breakdown," IMACS 1st International Conference on Computational Physics, University of Colorado, Boulder, June 1990, pp. 46-51. Also to appear in the Journal of Computer Physics Communications. North Holland Publishing Co., 1991.

16. Meadows, K., Kumar, A. and Hussaini, M., "A Computational Sudy on the Interaction Between a Vonex and a Shock Wave," AIAA 89-1043, April 1989. 


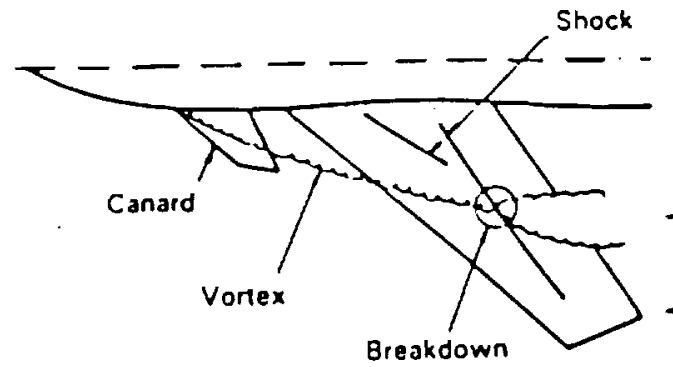

a. Transonic shock-vorex interaction on wings

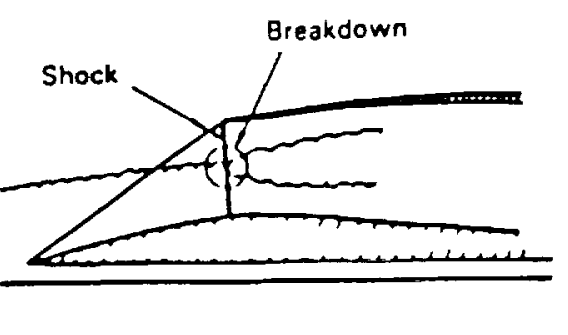

b. Supersonic shock-vorex interaction at inlets

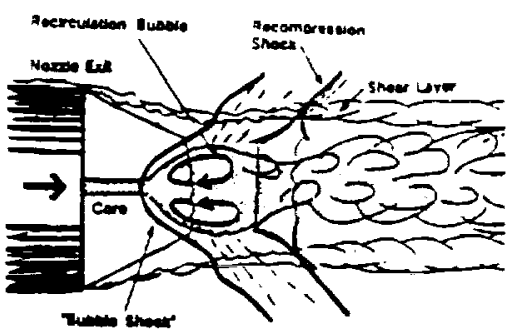

c. Supersonic shock-vorex interaction at nozzle exits

Figure 1. Applications of vorex-shock interaction and vortex breakdown
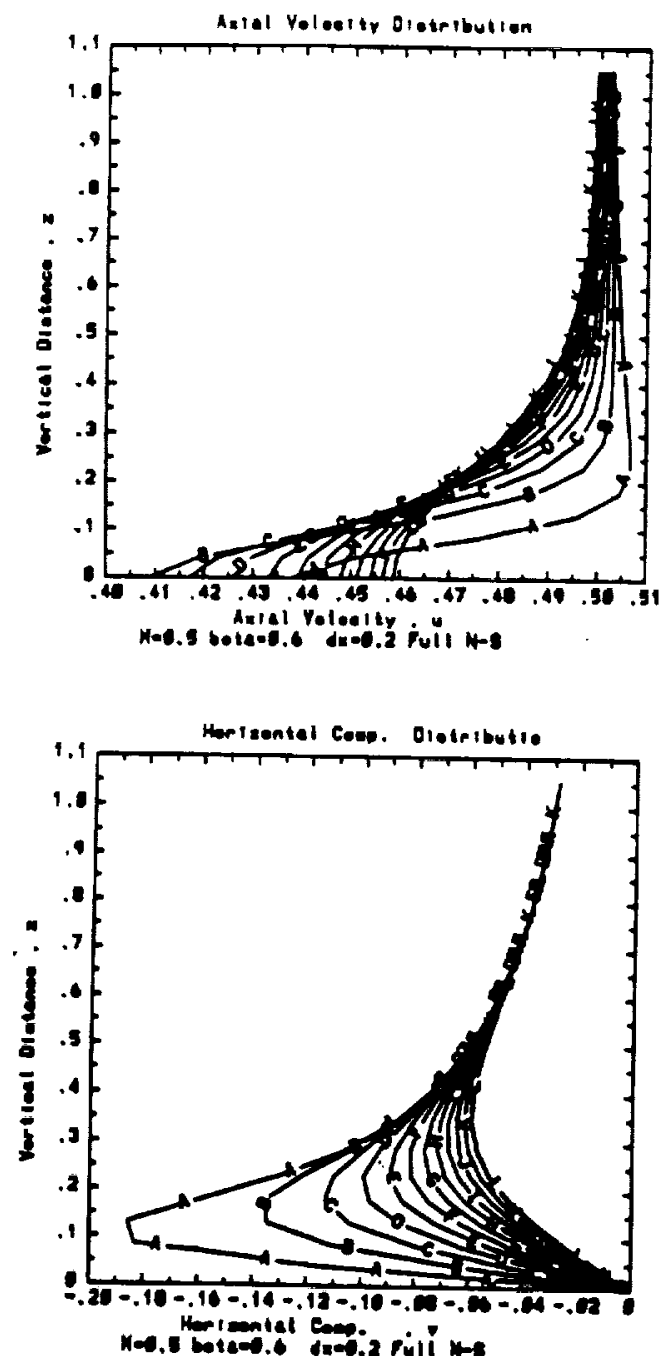
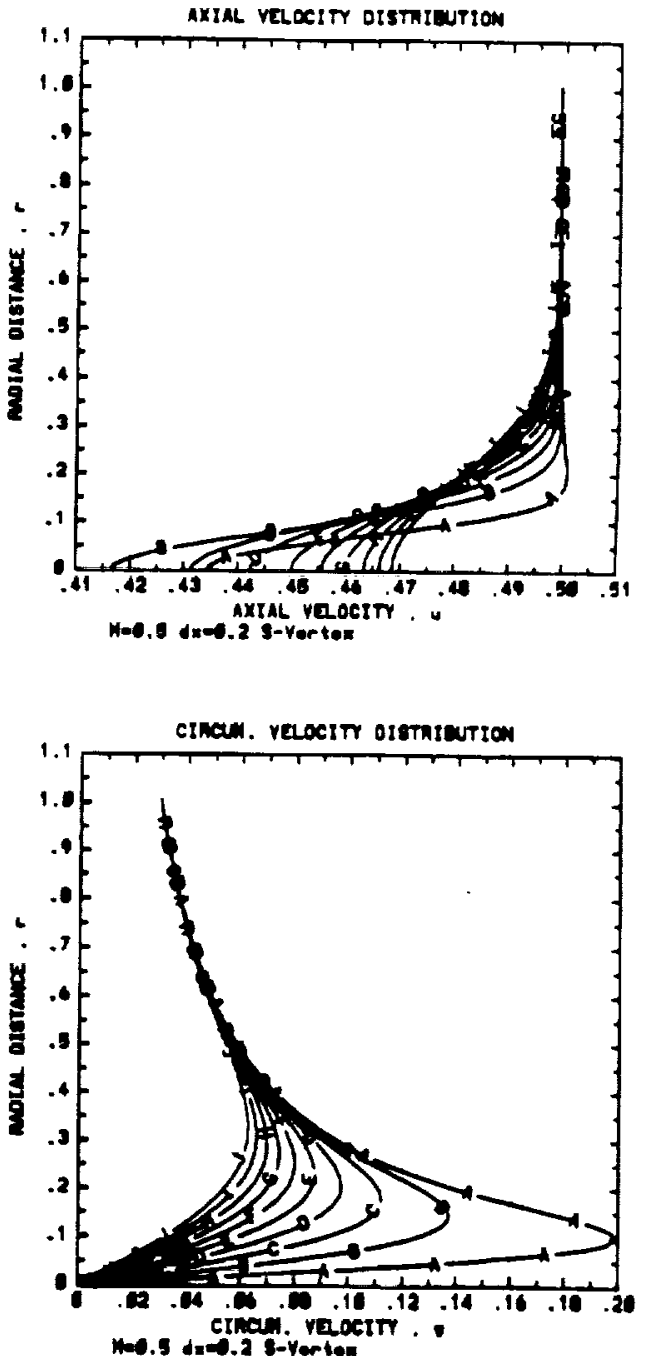

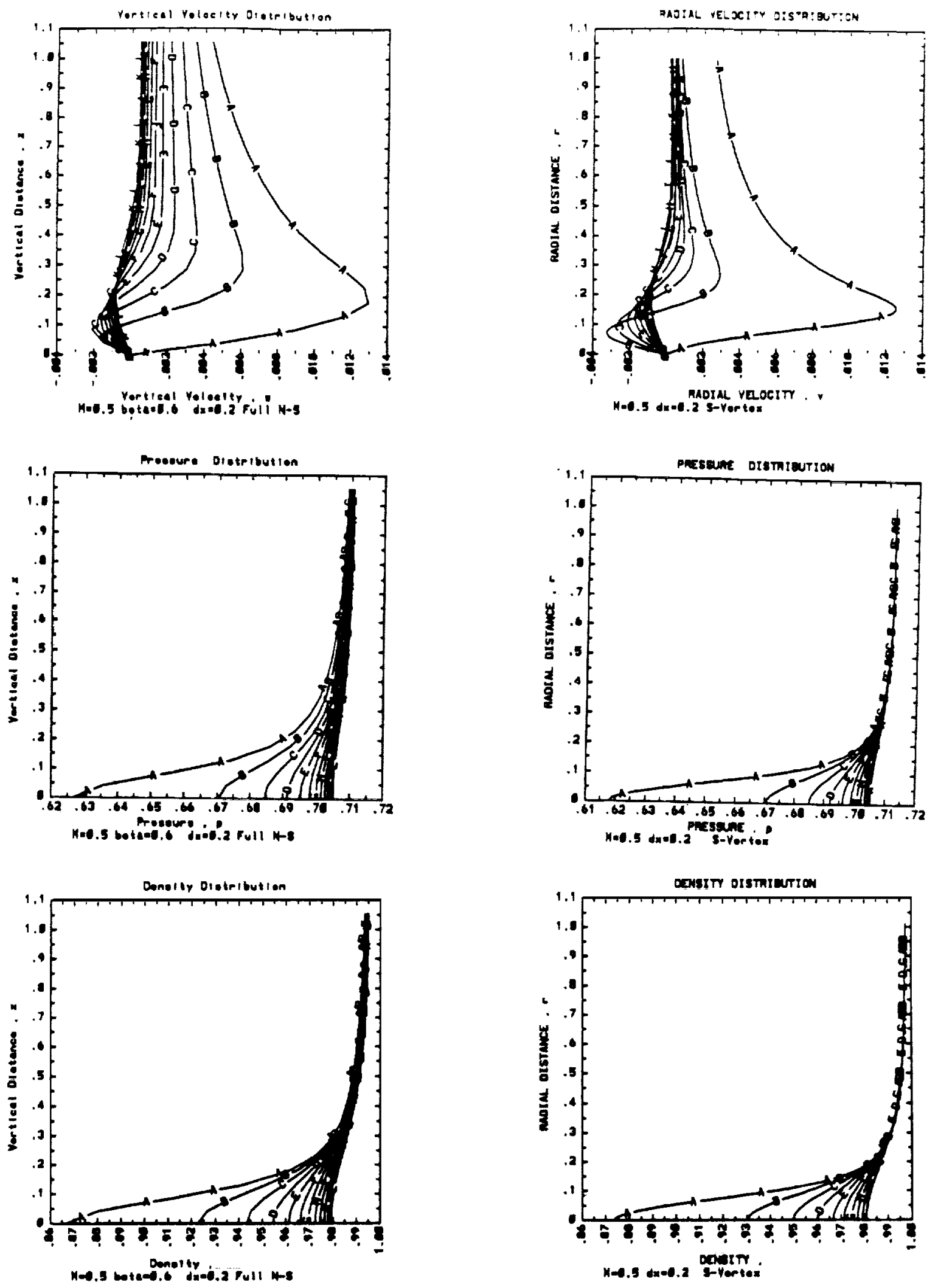

Navier-Stokes solutions

Slender-vortex solutions

Figure 2. Comparison of flow profiles using Navier-Stokes and slender-vortex equations, $\mathrm{M}_{\mathrm{e}}=0.5, \beta=0.6,\left(\frac{\mathrm{dp}}{\mathrm{dx}}\right)_{\mathrm{e}}=0,100 \times 51 \times 51$ grid for NS Eqs. 


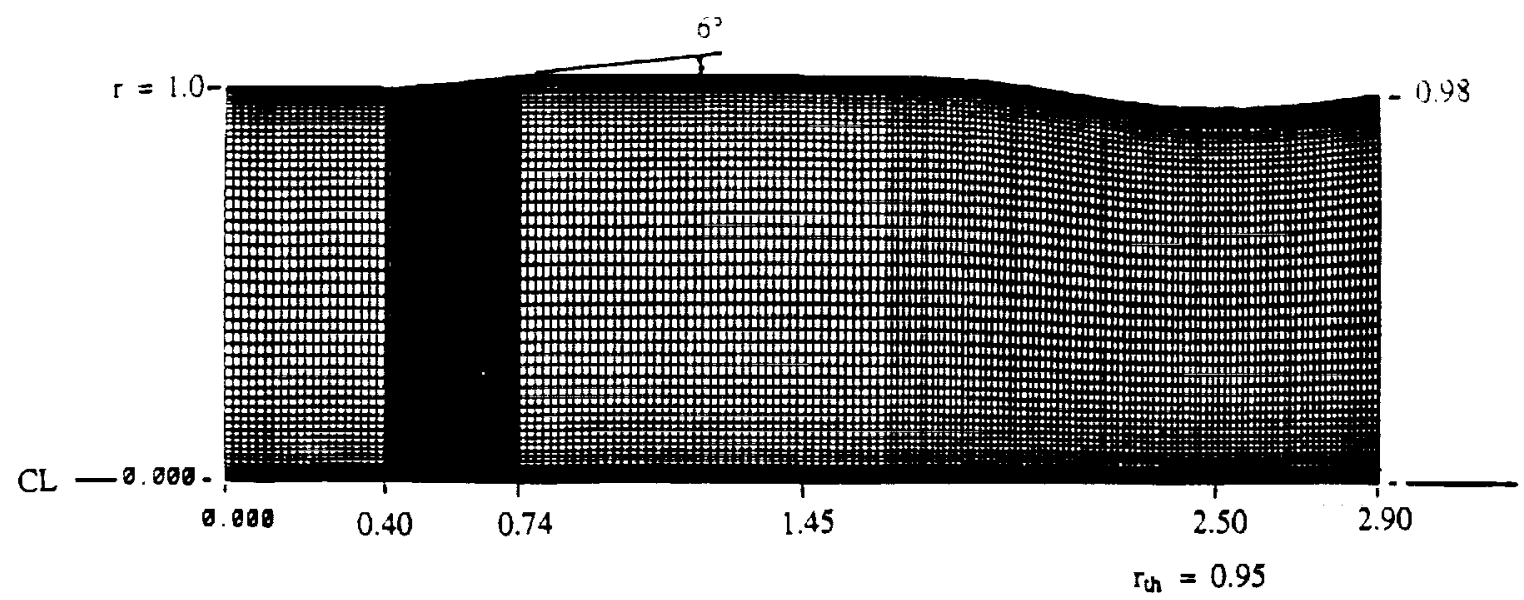

Figure 3. Typical grid for a supersonic axisymmetric duct configuration; $200 \times 51 \times 2$
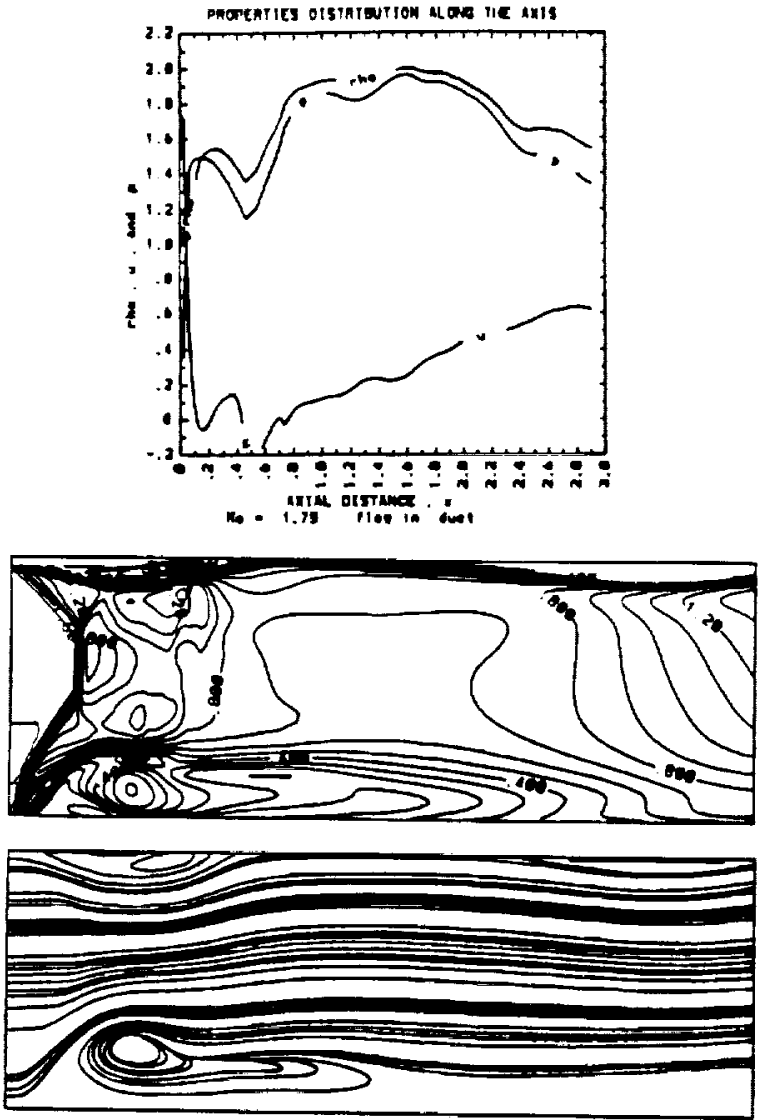

$(0.0,0.61)$

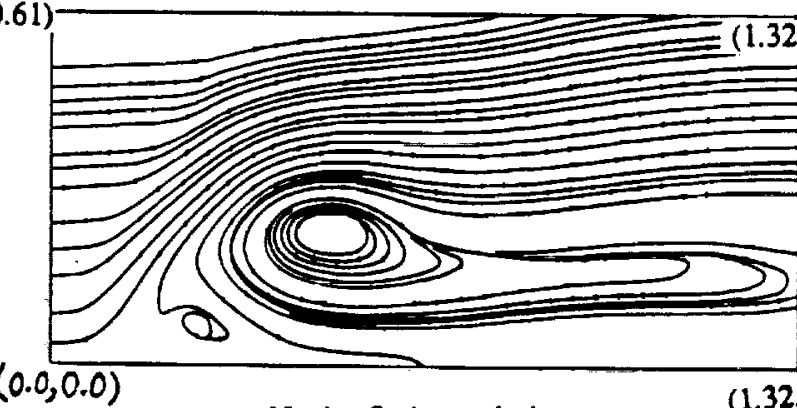

Navier-Stokes solutions
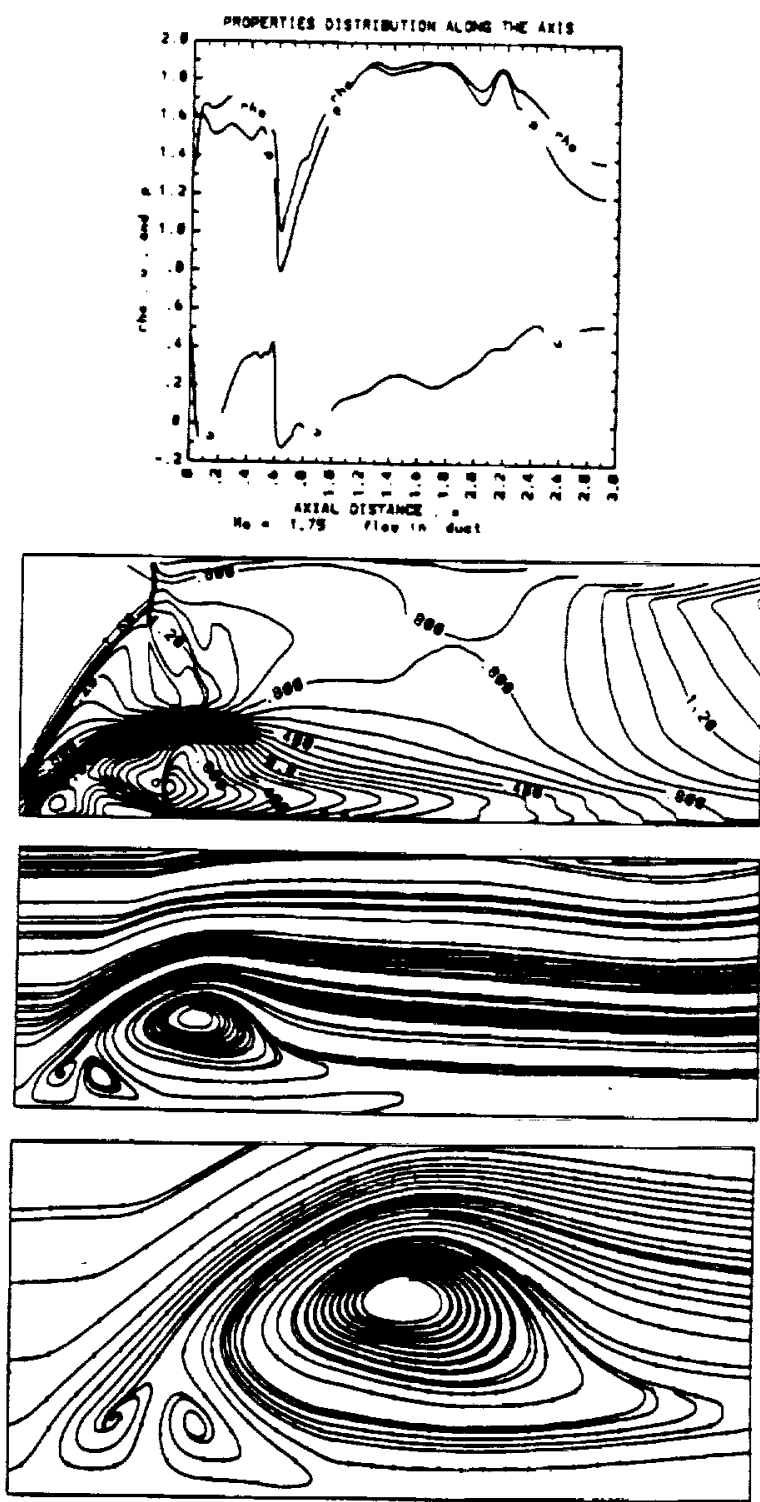

Euler solutions

Figure 4. Comparison of steady flow solutions using Navier-Stokes and Euler Eqs.; properties variation at centerline, Mach contours, sureamlines, blow-up at breakdown, $M=1.75$, $\beta=0.32, R_{0}=10^{4}$. 

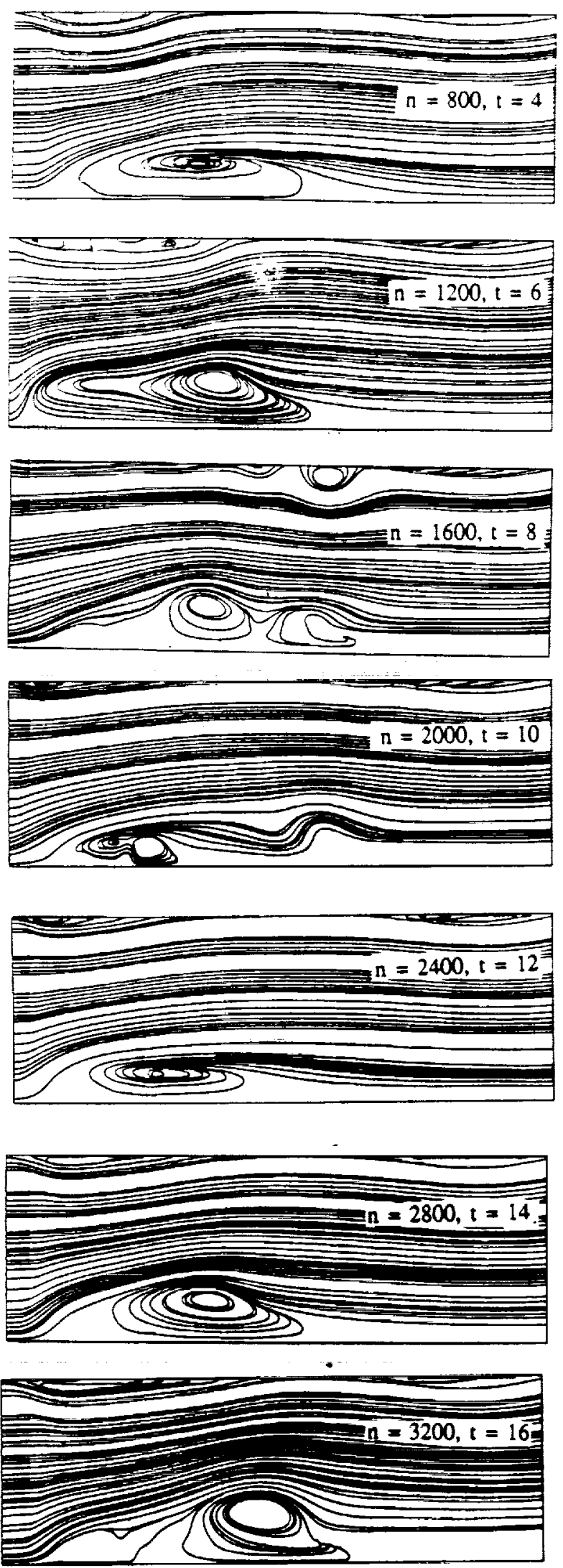

Navier-Stokes solutions
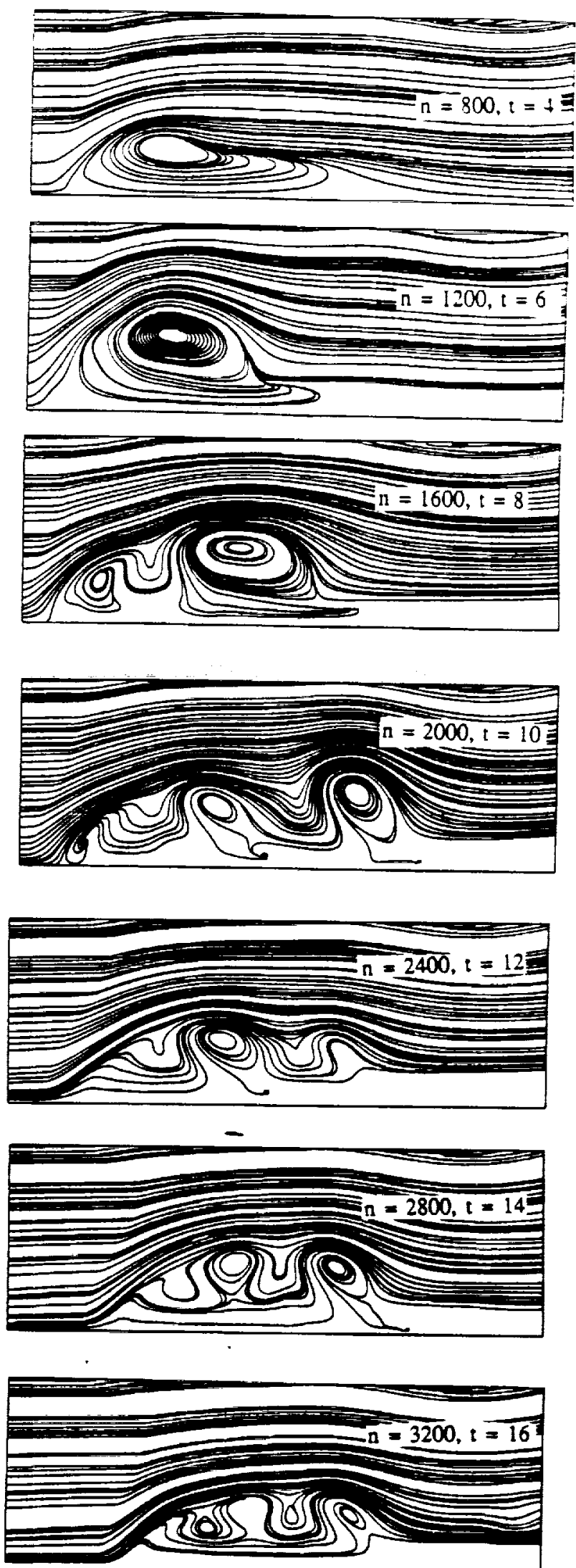

Euler solutions

Figure 5. Comparison of unsteady flow streamlines using Navier-Stokes and Euler Eqs., $M=$ $1.75, \beta=0.32, R_{3}=10^{4}, \Delta t=0.005$. 

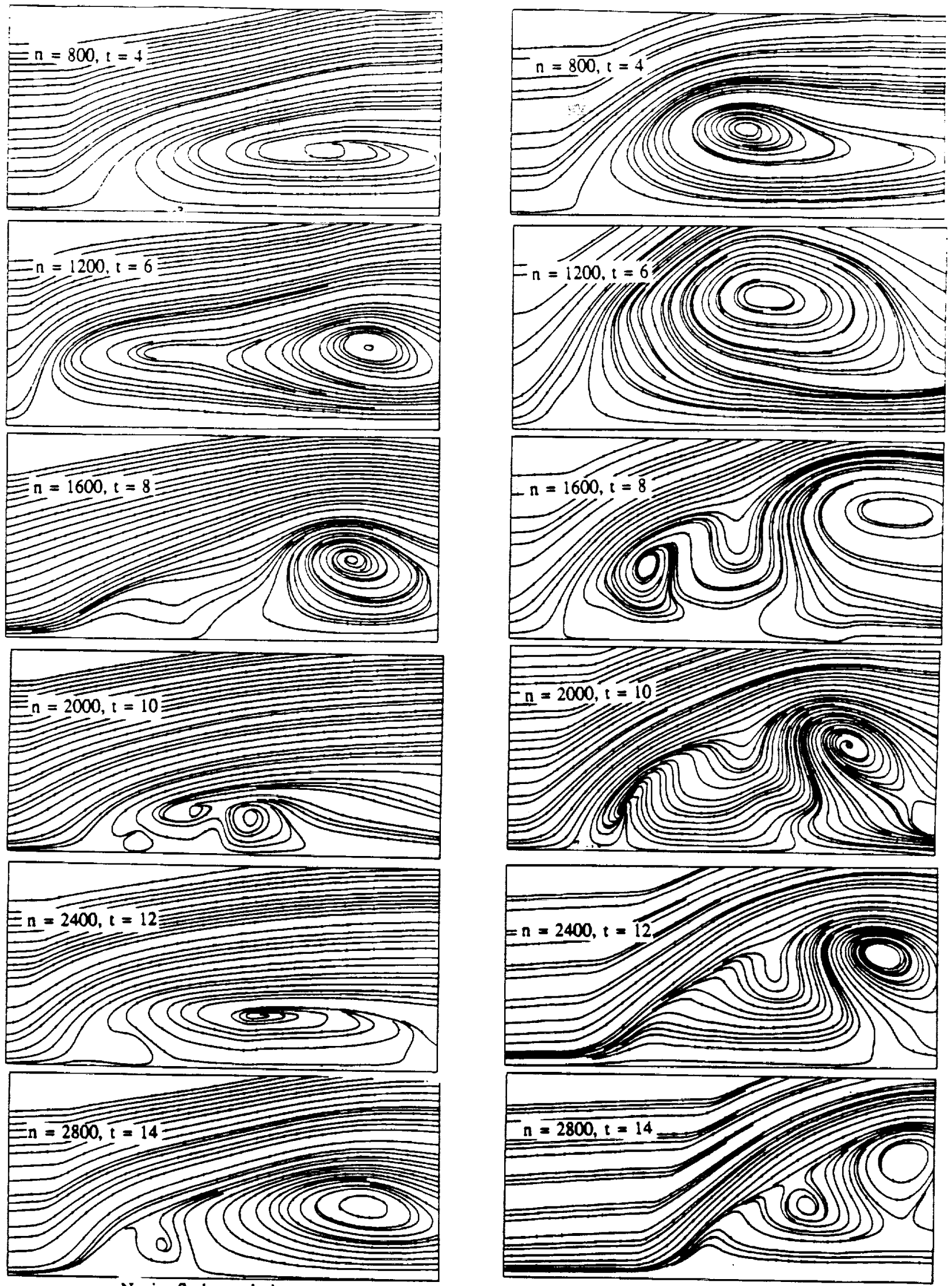

Navier-Stokes solutions

Euler solutions

Figure 6. Comparison of unsteady flow blow-up of sureamlines using Navier-Stokes and Euler Eqs., $M=1.75, \beta=0.32, R_{t}=10^{4}, \Delta t=0.005$. 

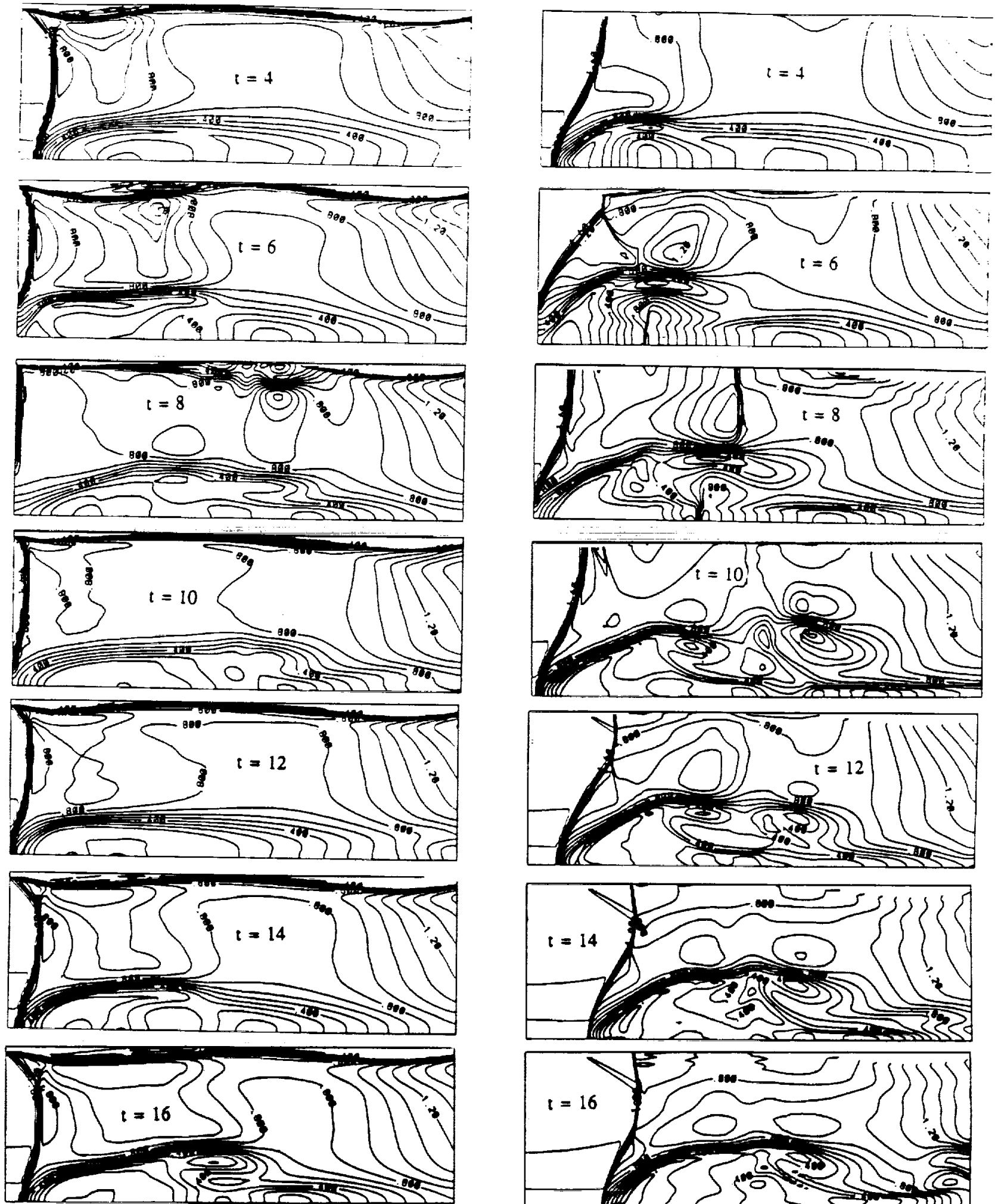

Navier-Stokes solutions
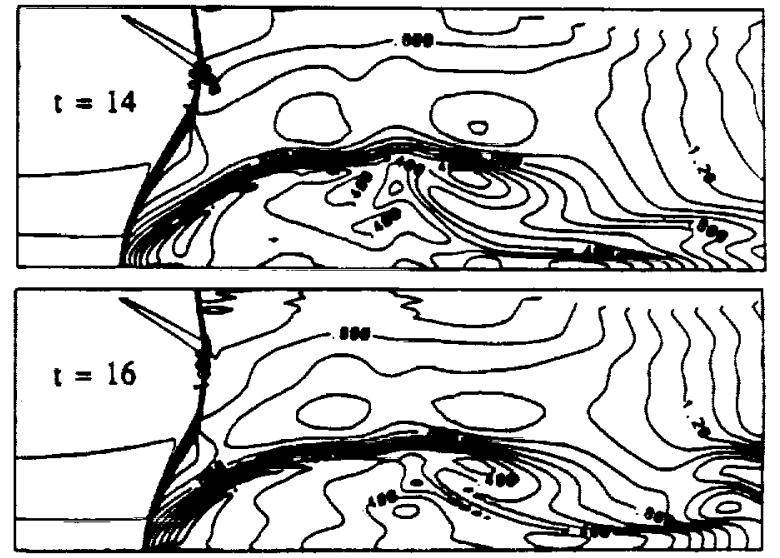

Euler solutions

Figure 7. Comparison of unsteady flow Mach contours using Navier-Stokes and Euler Eqs., M $=1.75, \beta=0.32, R_{e}=10^{4}, \Delta t=0.005$. 


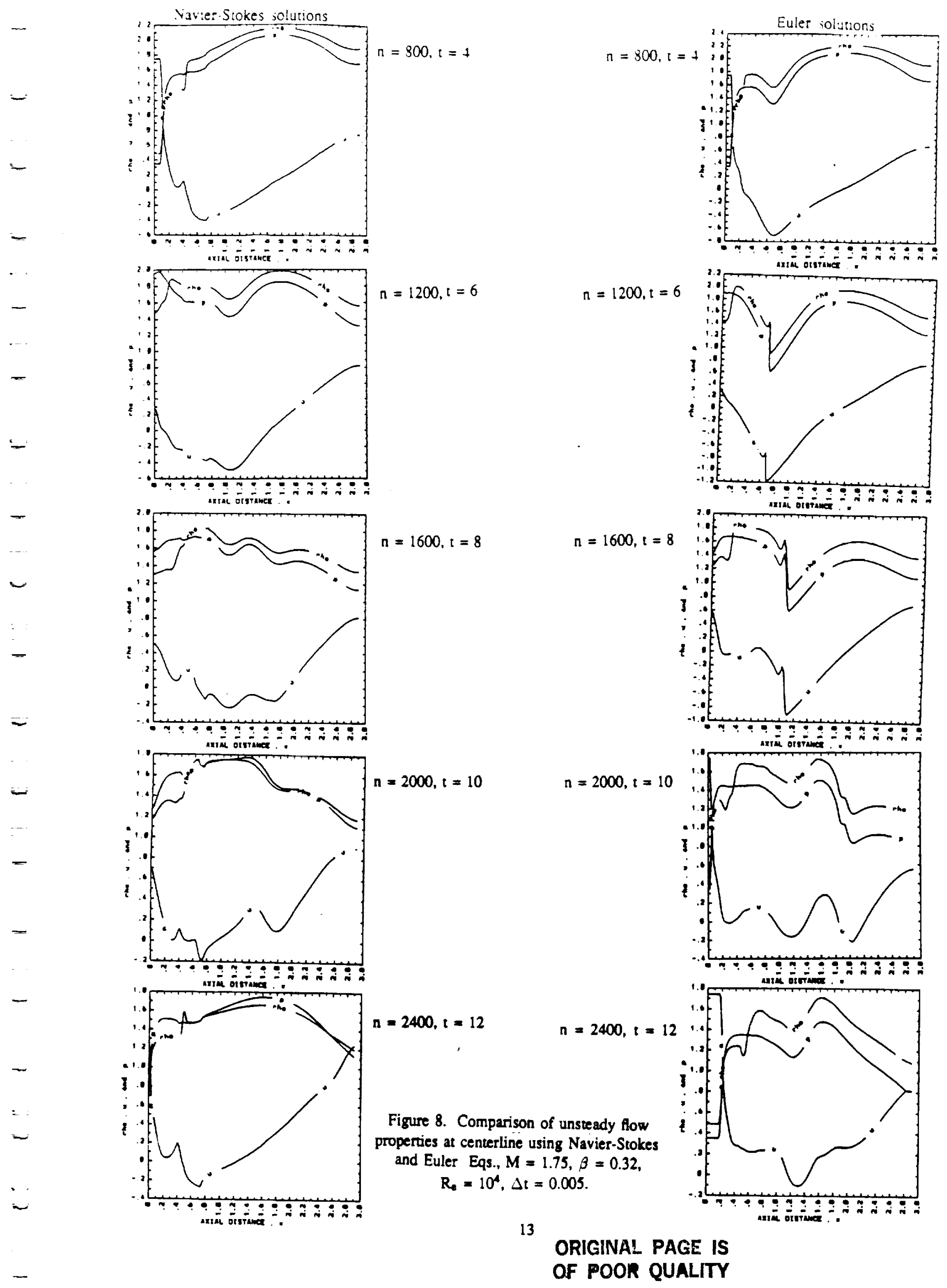



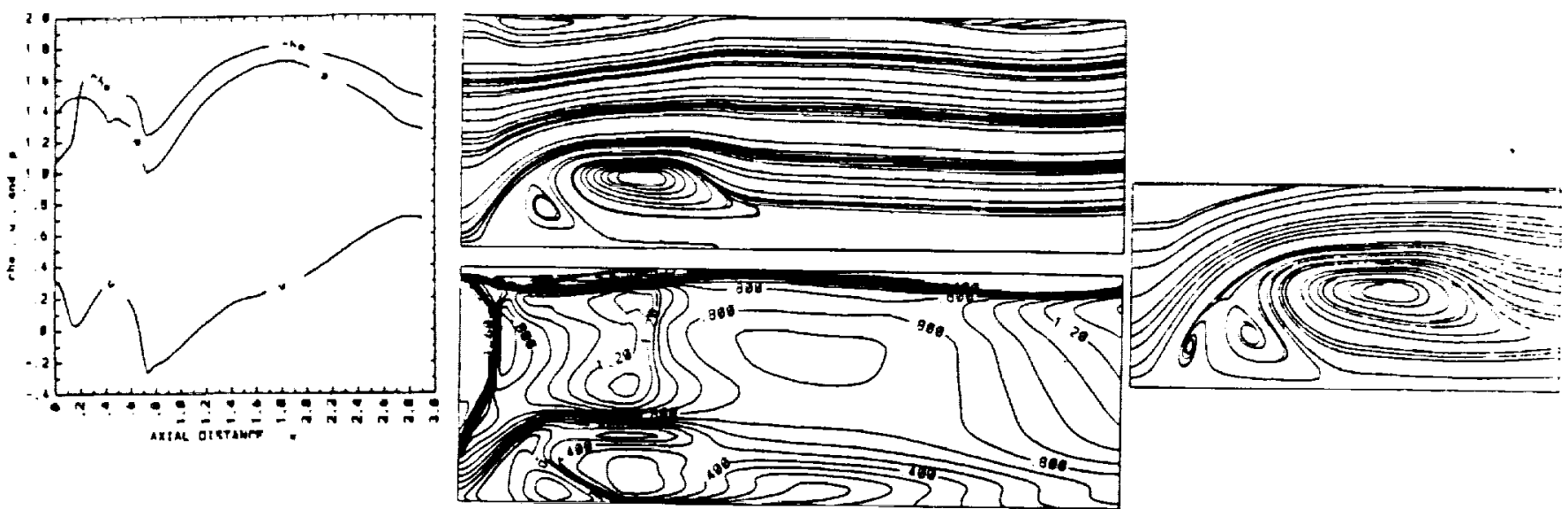

Figure 9. Steady flow solution using Navier-Stokes Eqs., properies at centerline, streamlines,
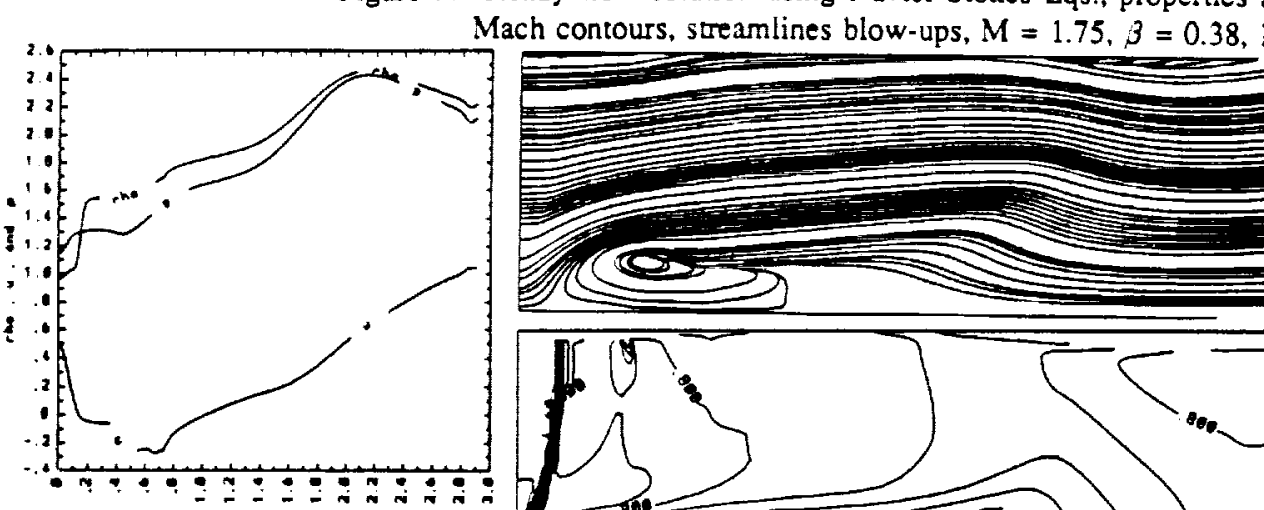

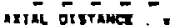
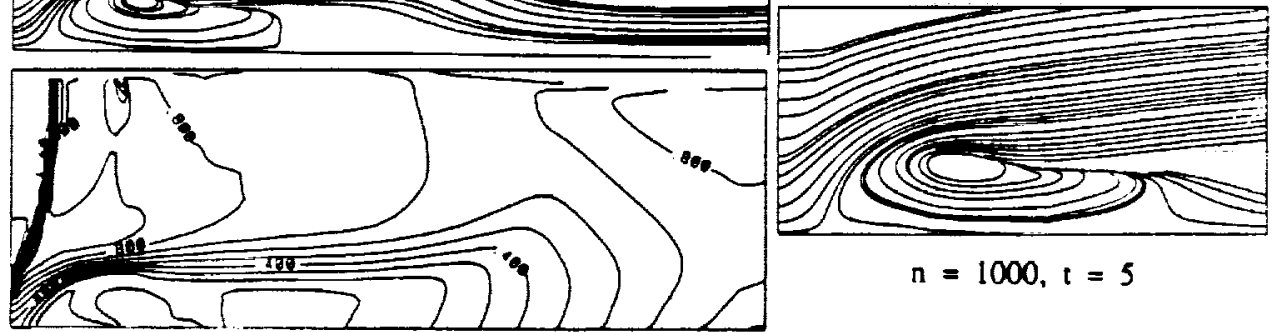

$n=1000, t=5$
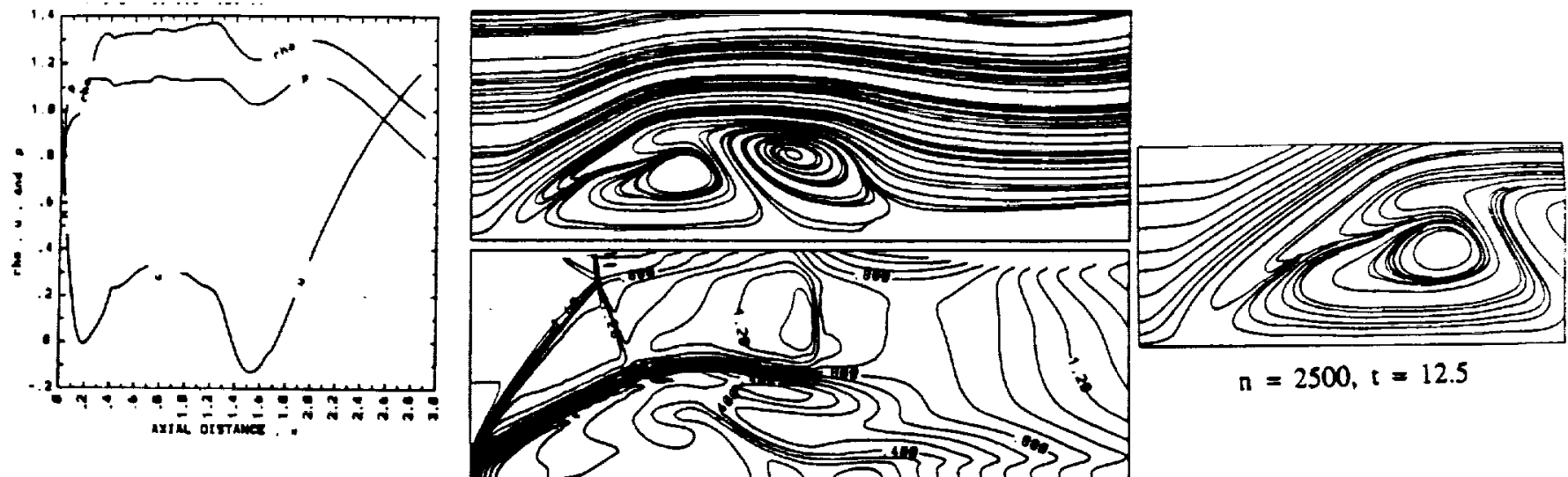

$n=2500, t=12.5$

$\underline{E}$

$\bar{E}$
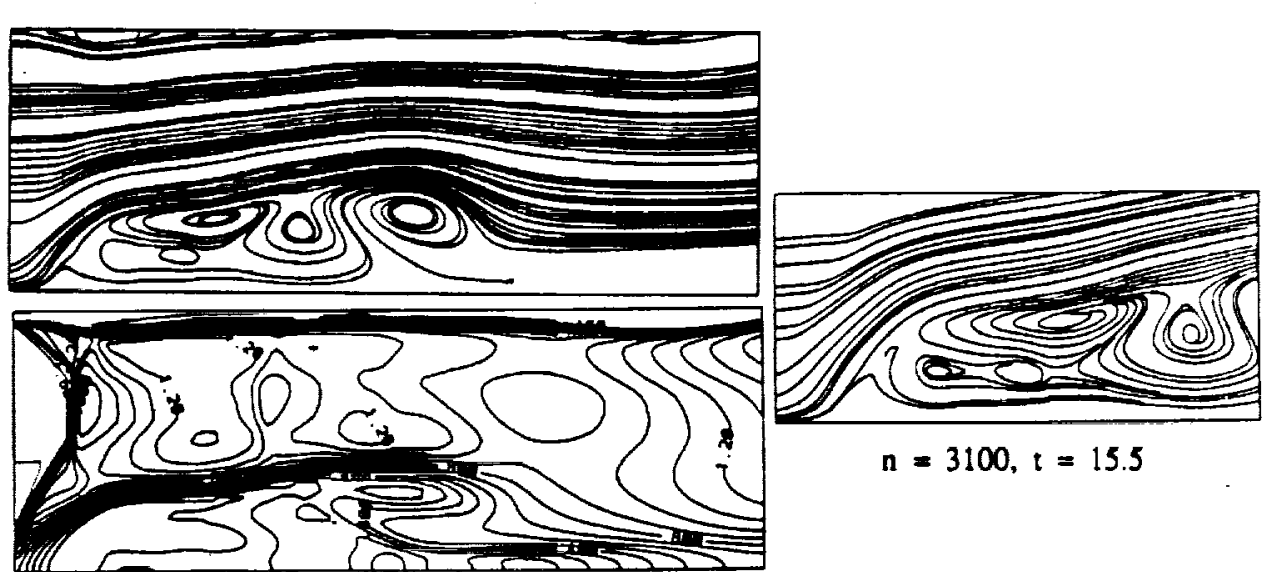

$\mathrm{n}=3100, \mathrm{t}=15.5$

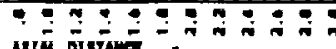

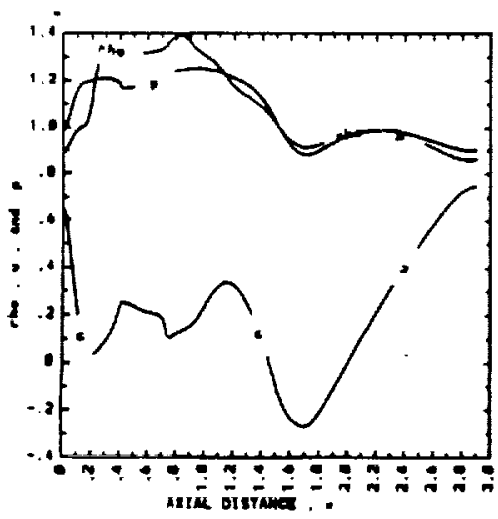

Figure 10. Unsteady flow solution using Navier-Stokes Eqs., properties at centerline, streamlines, Mach contours, streamlines blow-ups, $M=1.75, \beta=0.38, R_{\mathrm{a}}=10^{4}$. 